\title{
Vertical Structure of Radiative Heating Rates of the MJO during DYNAMO
}

\author{
Karen M. Shell, Simon P. De Szoeke, And Michael MaKiyama \\ Oregon State University, Corvallis, Oregon \\ ZHE FENG \\ Pacific Northwest National Laboratory, Richland, Washington
}

(Manuscript received 11 July 2019, in final form 3 December 2019)

\begin{abstract}
The vertical structure of radiative heating rates over the region of the tropical Indian Ocean associated with the MJO during the DYNAMO/ARM MJO Investigation Experiment is presented. The mean and variability of heating rates during active, suppressed, and disturbed phases are determined from the Pacific Northwest National Laboratory Combined Remote Sensing Retrieval (CombRet) from Gan Island, Maldives $\left(0.69^{\circ} \mathrm{S}\right.$, $73.15^{\circ} \mathrm{E}$ ). TOA and surface fluxes from the CombRet product are compared with collocated 3-hourly CERES SYN1deg Ed4A satellite retrievals. The fluxes are correlated in time with correlation coefficients around 0.9, yet CombRet time-mean OLR is $15 \mathrm{~W} \mathrm{~m}^{-2}$ larger. Previous work has suggested that CombRet undersamples high clouds, due to signal attenuation by low-level clouds and reduced instrument sensitivity with altitude. However, mean OLR differs between CombRet and CERES for all values of OLR, not just the lowest values corresponding to widespread high clouds. The discrepancy peaks for midrange OLR, suggestive of precipitating, towering cumulus convective clouds, rather than stratiform cirrus clouds. Low biases in the cloud-top height of thick clouds substantially contribute to the overestimate of OLR by CombRet. CombRet data are used to generate composite shortwave and longwave atmospheric heating rate profiles as a function of the local OLR. Although there is considerable variability in CombRet not directly related to OLR, the timeheight structure of mean heating rate composites generated using OLR as the interpolant is broadly representative of tropical convective variability on intraseasonal time scales.
\end{abstract}

\section{Introduction}

In the tropical troposphere, atmospheric radiative divergence-and hence the column-integrated heating rate-is largely determined by clouds. High-altitude clouds, and to a lesser extent water vapor, limit the emission of thermal radiation to space. High clouds also scatter sunlight back to space, reducing solar absorption and partially countering the thermal radiative warming. The net effect of deep convective cloud is to warm the troposphere, increasing the moist static energy (MSE) of the atmospheric column (e.g., Slingo and Slingo 1988; Raymond 2001; Tromeur and Rossow 2010). The Madden-Julian oscillation (MJO; Madden and Julian 1971) is a tropical eastward-propagating atmospheric pattern of alternating convectively active and suppressed phases, which operates on intraseasonal time scales. MSE anomalies, and hence deep convective

Corresponding author: Karen Shell, kshell@coas.oregonstate.edu clouds, influence the propagation of the MJO (Lin and Mapes 2004; Ma and Kuang 2011; Andersen and Kuang 2011; Yasunaga and Mapes 2012; Sobel et al. 2014).

Increases in MSE near the surface and radiative cooling of the upper troposphere decrease the stratification of the troposphere. Convection relieves the resulting instability, adjusting the thermal structure of the atmosphere to maintain a quasi-equilibrium (Neelin and Zeng 2000; Raymond 2001). Precipitation resulting from this convection can generate circulations that either increase or decrease tropospheric MSE. Because MSE has a minimum in the midtroposphere, upward velocities peaking in the lower troposphere import MSE, while vertical velocities that peak in the upper troposphere export MSE from the column (Neelin and Held 1987; Raymond 2001; Inoue and Back 2015). Gross moist stability (GMS; Neelin and Held 1987; Raymond et al. 2009) is a measure of the relationship between moist convection and the net column forcing of MSE. 
The weak planetary rotation of the tropics generates insufficient Coriolis force to balance significant pressure gradients. As a result, horizontal temperature gradients are weak (Sobel and Bretherton 2000). In the absence of horizontal temperature gradients, the temporal and spatial variability of deep convection is largely determined by moisture, whose variations affect column MSE without significantly affecting density (Raymond and Fuchs 2009; Sugiyama 2009). However, radiation also has a significant effect on the column MSE (Johnson et al. 2015; Del Genio and Chen 2015; Ciesielski et al. 2017). Decreased outgoing longwave radiation associated with deep cold convective clouds represents an anomalous source of column MSE. Reflection of solar radiation by the clouds is a sink of MSE, cancelling about half of the longwave effect. The radiative feedback of clouds to atmospheric column-integrated MSE is key for the maintenance of MSE anomalies associated with the MJO, with local sensible and latent surface fluxes perhaps playing a lesser role (Sobel et al. 2014).

In this study, we examine high temporal and spatial resolution radiative heating rate profiles for the DOE Atmospheric Radiation Measurement (ARM) site over Addu Atoll, Maldives, during the Dynamics of the MJO/ ARM Investigation Experiment (DYNAMO/AMIE) field campaign (Yoneyama et al. 2013). The DYNAMO/AMIE field campaign captured three deep convective events, two of which are associated with active phases of the MJO. The Pacific Northwest National Laboratory (PNNL) Combined Remote Sensing Retrieval (CombRet; Comstock et al. 2013; Feng et al. 2014) uses surface-based multiinstrument cloud remote sensing and sounding profiles, combined with radiative transfer modeling, to retrieve radiative heating rate profiles. Challenging these efforts, radiatively important high cirrus clouds are difficult to measure from the surface and are often significantly undersampled due to attenuation of sensor signals by lower clouds and rain (Protat et al. 2014). This undersampling leads to overestimated outgoing longwave radiation (OLR) in cloudy profiles and, hence, underestimation of the longwave cloud radiative effect (Feng et al. 2014).

OLR, readily observed by satellites, is the most varying component of the column radiative divergence. While active-wavelength remote sensing satellite products, such as CloudSat and Cloud-Aerosol Lidar and Infrared Pathfinder Satellite Observation (CALIPSO), provide retrievals of atmospheric heating rates (Del Genio and Chen 2015), more widely available passiveradiance satellite observations retrieve surface radiative properties indirectly through the atmosphere and do not provide high-resolution vertical heating rate profiles. We introduce a method that reproduces satellite OLR by design by combining OLR-composited groundbased CombRet vertical heating rate retrievals with the Clouds and the Earth's Radiant Energy System (CERES; Wielicki et al. 1996) OLR time series. The derived radiative heating rates are representative of means on intraseasonal time scales.

This methodology can generate vertical profiles of heating rates from an OLR time series in similar locations where no detailed ground-based remote sensing is available. The central Indian Ocean location of Gan is of particular interest because it is at this longitude that the MJO typically first reaches its full amplitude. Certainly, one should not overinterpret the results from Gan Island. Changes in the water vapor and cloud population differ seasonally and geographically throughout the warm pool, but the intraseasonal variations are similar throughout the austral spring-summer from the central Indian Ocean to the west Pacific. Furthermore, differences in sampling between an ARM station and the CERES satellite are not unique to any location, and changes in clouds and water vapor are likely to have the same effect on radiation throughout the warm pool. The intraseasonal composite cloud and water vapor structure at Gan agrees with that over multiple stations across the warm pool.

We begin by describing the data and methods used in this work (section 2). Section 3 discusses the mean CombRet radiative heating profiles for the convectively suppressed, disturbed, and active periods during the DYNAMO/AMIE field campaign. In section 4, we compare radiative fluxes inferred from satellite by CERES Edition 4 with fluxes from CombRet. Section 5 presents the OLR-composite-mean heating rates, along with the method for generating OLR-consistent radiative heating rates from a satellite OLR time series, and applies this methodology to produce vertically resolved heating rates that agree with CERES OLR. The conclusions are presented in section 6 .

\section{Data and methods}

The AMIE-Gan/DYNAMO campaign collected data from Gan Island, Maldives $\left(0.69^{\circ} \mathrm{S}, 73.15^{\circ} \mathrm{E}\right)$, from 10 October 2011 to 8 February 2012, covering two convectively active MJO events and one other deep convective event. The PNNL CombRet (Feng et al. 2014) provides data for this 121-day period. CombRet combines S-band and Ka-band radar data and micropulse lidar data to estimate profiles of cloud microphysical properties such as liquid/ice water content and effective particle size. Subsequently, CombRet applies the cloud properties, along with surface measurements and atmospheric sounding profiles, to a radiative transfer model to 
calculate radiative heating rate profiles. The 30 -s data are averaged to 3-hourly resolution. This retrieval is the same as that used in Ciesielski et al. (2017). Unless otherwise stated, we use the 3-hourly CombRet data. We use the daily Real-time Multivariate MJO index (RMM; Wheeler and Hendon 2004) to classify each day of the observational period into the convectively suppressed (RMM phases 4-8), convectively disturbed (phase 1 ), or convectively active (phases 2-3) phase over the central equatorial Indian Ocean.

We compare results from the CombRet product with CERES (Wielicki et al. 1996; Minnis et al. 2011a,b; Doelling et al. 2018) SYN1deg data (CERES SYN1deg3 Hour Ed4A) for the observational period. CERES Edition4A SYN1deg products leverage algorithm improvements included in the Edition 4 suite of CERES radiance and swath products. Since earlier work (e.g., Johnson et al. 2015; Ciesielski et al. 2017) compared CombRet data with an earlier version of the SYN1deg data (Ed3A), we perform comparisons with this dataset as well to determine the sensitivity of the conclusions to the CERES version.

The SYN1deg product provides two sets of radiative fluxes on a $1^{\circ} \times 1^{\circ}$ grid. The observed top-of-atmosphere (TOA) fluxes are directly measured by CERES. The computed TOA, surface, and atmospheric fluxes are produced by tuning input satellite-derived cloud and aerosol properties, atmospheric reanalysis, and surface data within a radiative transfer model to better match the observed CERES TOA fluxes. We use computed CERES 3-hourly mean TOA and surface radiative flux densities for comparison with CombRet, but observed CERES OLR as the interpolant in section 5. We average over 12 grid points, corresponding to the equatorial region near Gan Island $\left( \pm 2^{\circ} \mathrm{N}, 72^{\circ}-75^{\circ} \mathrm{E}\right)$.

To estimate the effects of clouds on these vertical profiles, we calculate the cloud radiative effect (CRE) by subtracting the clear-sky from the all-sky radiative heating rates or fluxes. In CombRet, the clear-sky values are calculated for each time step by performing a second radiative transfer calculation with the same atmospheric conditions, but cloud fraction set to zero (i.e., the "cloud-removed" clear-sky fluxes; Kato et al. 2013). Differences in CRE between two MJO phases provide estimates of the radiative effects of differences in cloud properties between the phases. However, it is possible that changes in surface and atmospheric properties other than clouds (e.g., water vapor, temperature) are contributing somewhat to CRE differences, since these property changes may affect all-sky and clear-sky fluxes differently (Soden et al. 2004).

In contrast to CombRet, many satellite-derived clearsky fluxes are determined by averaging fluxes using only cloud-free pixels. For example, the observed CERES clear-sky fluxes are calculated using cloud-free footprints within each $1^{\circ}$ by $1^{\circ}$ region. Since water vapor tends to be lower in clear-sky pixels compared with cloudy pixels, this difference in methodologies would result in a dry bias of the observed CERES clear-sky fluxes compared to CombRet clear-sky fluxes, which in turn, could lead to an overestimate of the longwave CRE of roughly 10\% (Sohn et al. 2010; Kato et al. 2013). For this reason, we use the computed CERES clear-sky fluxes, which are calculated using the "cloud-removed" method, consistent with the CombRet methodology.

\section{CombRet vertical radiative heating profiles}

Recent work has shown the importance of the column net radiative heating as a positive feedback to moist static energy (MSE) anomalies (Sobel et al. 2014; Johnson et al. 2015; Del Genio and Chen 2015; Ciesielski et al. 2017). We begin by examining the vertically resolved CombRet radiative heating rates, similarly to Fig. 10 in Johnson et al. (2015), but with the longwave (LW) and shortwave (SW) components treated separately. Figures $1 \mathrm{a}$ and $1 \mathrm{~b}$ show the 3 -hourly heating rates over the observational period. Overlaid on each plot in yellow are the Wheeler and Hendon (2004) MJO phases. The light blue circles indicate convectively active days (RMM phases 2-3), with enhanced LW cooling and SW warming in the upper troposphere, and decreased LW cooling and SW warming in the midtroposphere. In contrast, convectively suppressed days (RMM 4-8) show less vertical variability in LW cooling and SW heating. However, the large variability in the 3-hourly data makes it difficult to see the evolution of the heating rates with $\mathrm{MJO}$ cycles.

To better visualize this evolution, we average all the days in the convectively suppressed (red; RMM 4-8), convectively disturbed (purple; RMM 1), and convectively active (blue; RMM 2-3) phases (Fig. 2). These figures are similar to those in Fig. 9 in Ciesielski et al. (2017), but with three phase classifications (suppressed, disturbed, and active) compared to their four classifications (suppressed, bottom heavy, deepconvective, and stratiform). The light and dark shadings indicate one standard deviation around the mean and the standard error of the mean, respectively, based on daily-average values. CombRet overestimates retrieved water content around $5 \mathrm{~km}$ due to melting ice particles (Feng et al. 2014). The highly reflective liquid shells enhance radar reflectivity, creating a "bright band" that biases the results of the radiative transfer model. Hence, we omit heating rates at $600 \mathrm{hPa}$ to exclude melting-level artifacts from Fig. 2. 

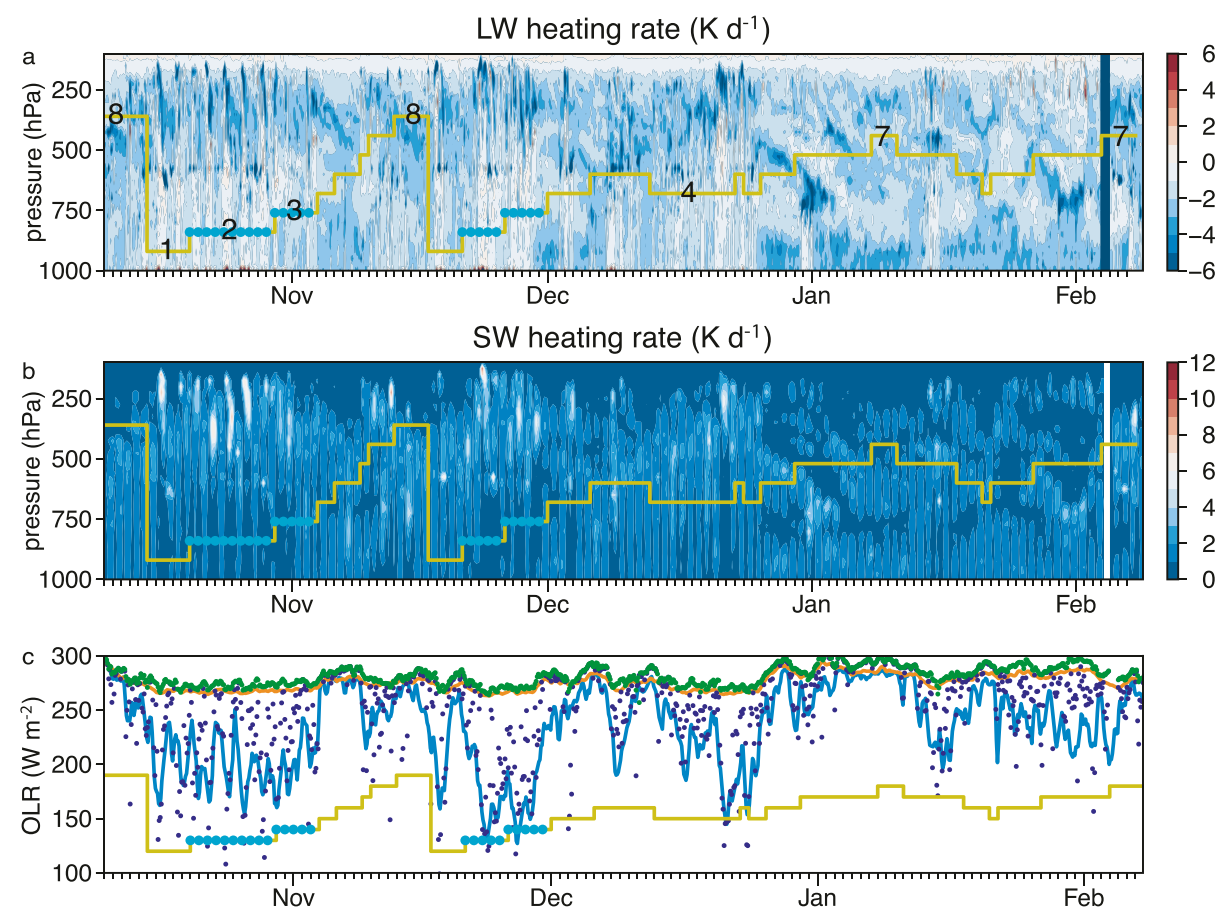

FIG. 1. (a) Longwave and (b) shortwave atmospheric radiative heating rate $\left(\mathrm{K} \mathrm{day}^{-1}\right)$ time-height series from the DYNAMO/AMIE Gan Atmospheric Radiation Measurement (ARM) CombRet retrieval. In (b), the SW heating rate is shown only for daytime scenes (five per day), and the magnitudes are multiplied by 5/8 for display purposes. (c) CERES Ed4 (solid) and CombRet (dots) outgoing longwave radiation (OLR; blue) and CERES Ed4 (solid red) and CombRet (green dots) clear-sky OLR, in $\mathrm{W} \mathrm{m}^{-2}$. The daily phase of the Real-time Multivariate MJO index (RMM; Wheeler and Hendon 2004) is indicated by the yellow lines; phases 2 and 3 (active phases) are highlighted with light blue circles.

The mean LW all-sky cooling decreases (i.e., a warming effect) in the lower troposphere and increases in the upper troposphere as the MJO phase transitions from the suppressed (4-8) to the active (2-3) MJO phases (Fig. 2a). Shortwave heating decreases from the suppressed to the active phase below $700 \mathrm{hPa}$ and increases above $500 \mathrm{hPa}$ (Fig. 2b).

To estimate the radiative effects of changes in clouds and noncloud constituents (e.g., water vapor and temperature) with MJO phase, we examine clearsky heating rates (Figs. 2c,d) and cloud radiative effects (Figs. 2e,f). For heights where the mean clear-sky heating rates (solid lines in Figs. 2c,d) are similar across phases, we infer that the differences in the mean all-sky fluxes associated with the MJO (Figs. 2a,b) are primarily due to clouds. This is the case for both the LW and SW clear-sky heating rates near the tropopause and SW heating in the midtroposphere. However, mean clear-sky heating rate in the upper troposphere is somewhat dependent on MJO phase. Here, active phases tend to have enhanced clear-sky LW cooling and SW heating, compared to the suppressed phases, by up to $0.6 \mathrm{~K} \mathrm{day}^{-1}(30 \%)$ and $0.4 \mathrm{~K} \mathrm{day}^{-1}(50 \%)$, respectively. The active phases correspond to increased temperature in the upper troposphere, slightly decreased temperature in the lower troposphere, and increased specific humidity throughout the troposphere, as shown in Ciesielski et al. (2017, their Fig. 10) and Del Genio and Chen (2015, their Figs. 6 and 7). These atmospheric profile changes lead to enhanced clear-sky LW emission in the upper troposphere and reduced upwelling LW radiation from below, resulting in enhanced upper tropospheric LW cooling in the active phase. Increased SW absorption (due to increased water vapor) enhances upper troposphere clear-sky SW heating. In the lower troposphere, the situation is reversed, with reduced clear-sky LW cooling, due to enhanced absorption and a reduced emission temperature, and reduced clear-sky SW warming, due to reduction of downwelling SW radiation, in the disturbed and active phases compared with the suppressed phase.

The suppressed phase exhibits larger day-to-day clear-sky LW and SW heating rate variability than the active phase (light shading in Figs. 2c,d). During the active phase, the troposphere is often close to saturation, given convection and the availability of moisture. 

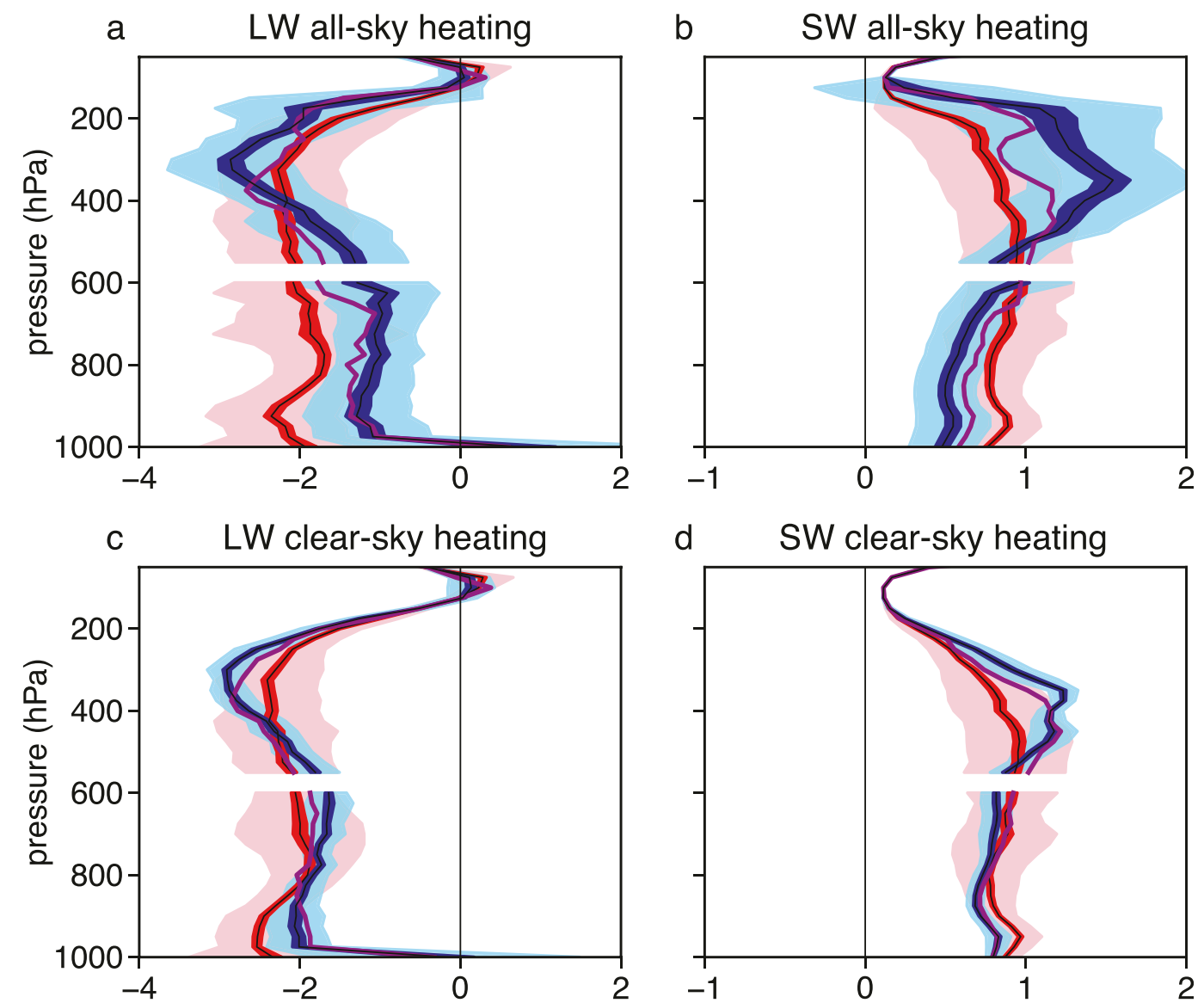

d SW clear-sky heating
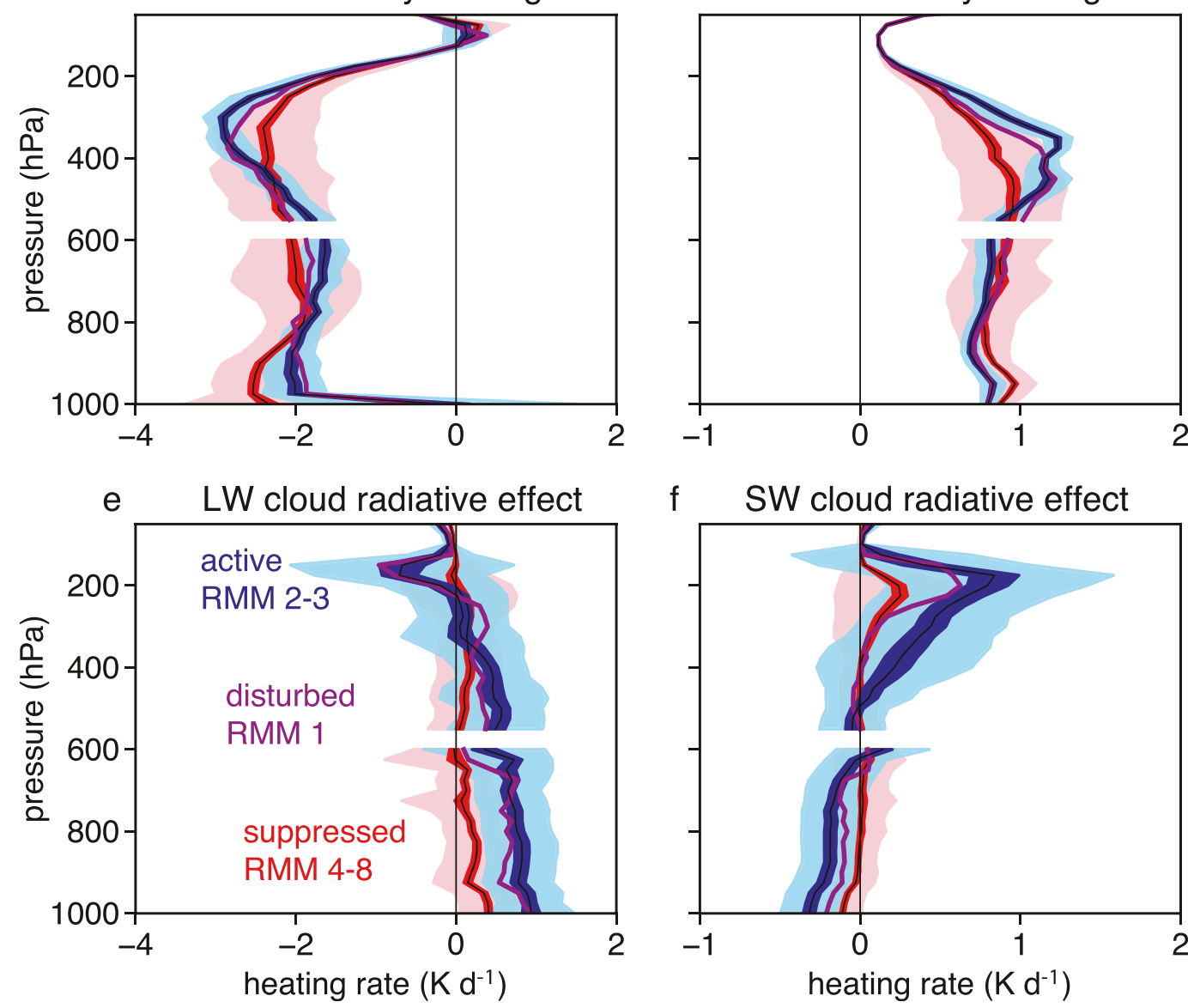

FIG. 2. Vertical profiles of (a),(b) all-sky, (c),(d) clear sky, and (e),(f) cloud radiative effect (left) LW and (right) SW radiative heating rates $\left(\mathrm{K} \mathrm{day}^{-1}\right)$ for the AMIE-Gan site, from the CombRet retrieval (Feng et al. 2014) for the convectively suppressed (red; RMM phases 4-8), convectively disturbed (purple; phase 1), and convectively active (blue; phases 2-3) phases of the RMM. Lines correspond to means, dark shading to plus or minus one standard error of the daily average, and light shading plus or minus one standard deviation of the daily average. The standard deviations and standard errors are omitted for the intermediate disturbed phase. 
TABLE 1. Time-mean, vertically averaged CombRet heating rates $\left(\mathrm{K} \mathrm{day}^{-1}\right)$ for the troposphere (up to $100 \mathrm{hPa}$ ) for the entire observational time period and from suppressed, disturbed, and active MJO days.

\begin{tabular}{lrcrr}
\hline \hline & Entire & Suppressed & Disturbed & Active \\
\hline LW all sky & -1.74 & -1.84 & -1.59 & -1.41 \\
LW clear sky & -1.94 & -1.97 & -1.89 & -1.84 \\
LW CRE & 0.19 & 0.12 & 0.30 & 0.43 \\
SW all sky & 0.79 & 0.77 & 0.83 & 0.87 \\
SW clear sky & 0.75 & 0.74 & 0.79 & 0.79 \\
SW CRE & 0.04 & 0.03 & 0.04 & 0.08 \\
Net (LW + SW) all sky & -0.95 & -1.07 & -0.76 & -0.54 \\
\hline
\end{tabular}

Since temperature changes are small, these active phase days have fairly similar specific humidities and, thus, similar clear-sky heating profiles. In contrast, during the suppressed phase, the troposphere is occasionally saturated, but usually it is not. The wider variability of relative humidity (and, hence, water vapor) increases the clear-sky radiative heating rate variability in the suppressed phase.

Cloud radiative effects (Figs. 2e,f) tend to peak higher than the clear-sky heating rates. CRE is sensitive to high clouds, while clear-sky rates are more sensitive to midlevel moisture. In agreement with Ciesielski et al. (2017), CRE is reduced during the suppressed phase (red lines), when cloud fraction is low, and amplified during the active phase (blue lines), when deep convective clouds strongly influence the atmospheric heating rate. Active phase LW CRE is positive throughout most of the troposphere, reaching $1 \mathrm{~K} \mathrm{day}^{-1}$ near the surface, with cooling near the tropopause. Active phase SW CRE is positive (up to $1 \mathrm{Kday}^{-1}$ ) in the upper troposphere, due to enhanced absorption associated with higher clouds, and somewhat negative in the lower troposphere, due to enhanced shading of lower levels. SW CRE peaks slightly higher and at more than twice the magnitude in the active phase compared to the suppressed phase. This agrees with the expectation that suppressed phases tend to have fewer and somewhat lower high clouds. The active phase tends to show greater variability of CRE.

Table 1 summarizes the vertically averaged (pressureweighted) tropospheric heating rates. The mean tropospheric cooling rate is $0.95 \mathrm{~K} \mathrm{day}^{-1}$, in agreement with the CombRet tropospheric cooling rate $\left(\sim 1 \mathrm{~K} \mathrm{day}^{-1}\right)$ reported by Johnson et al. (2015, p. 611). During the active phase, the magnitude of the mean net cooling rate drops to $0.54 \mathrm{~K} \mathrm{day}^{-1}$, while the suppressed phase has almost double the cooling of the active phase $\left(1.07 \mathrm{~K} \mathrm{day}^{-1}\right)$. Our analysis using the CombRet retrieval corroborates the results of Sobel et al. (2014), Johnson et al. (2015), Del Genio and Chen (2015), and
Ciesielski et al. (2017) that column net radiative heating acts as a positive feedback to intraseasonal MSE anomalies. Both the LW and SW heating rates contribute to this warming (i.e., the active phase has both enhanced SW warming and decreased LW cooling), but the additional SW absorption contributes only about $20 \%$ of the net heating difference.

\section{Comparison of CombRet with CERES data}

The AMIE-Gan field site provides high temporal and vertical resolution data for one location, yet the MJO pattern has a large horizontal extent, from the western Indian Ocean to the central tropical Pacific Ocean. Data from one location over a few MJO cycles are thus not necessarily representative of all MJO behavior. Satellite data provide near-global coverage and longer records, but not the high-resolution data necessary to fully quantify important processes.

Merging TOA flux information from CERES with surface observations from Gan provides a more representative picture of the radiative heating budget throughout the atmosphere. We compare Gan CombRet radiative fluxes to collocated CERES SYN1deg fluxes to test the similarity of radiative heating/cooling and cloud effects throughout the MJO cycle. Johnson et al. (2015) and Ciesielski et al. (2017) perform similar comparisons of CombRet and CERES SYN1deg Ed3 radiative heating rates. Here, we extend this comparison to the new version of CERES SYN1deg, Ed4. We evaluate the possibility of underrepresentation of high clouds, a common problem with ground-based datasets (Protat et al. 2014), for the CombRet retrieval. Additionally, we explore which aspects of the column properties in CombRet and CERES result in the discrepancies in radiative fluxes.

\section{a. Mean flux differences and correlations}

Table 2 provides time-mean TOA and surface fluxes for the CERES Ed4 and CombRet datasets. The two datasets are correlated in time, with correlation coefficients for daily surface and TOA fluxes ranging from 0.84 to 0.98 (excluding upwelling surface $\mathrm{SW}$, which is of small magnitude). The high temporal correlation between CERES and CombRet data suggests that anomalies in the CombRet station data are representative of anomalies in the larger region, despite their differences in sampling. While the two datasets are correlated in time, they have mean differences. CombRet OLR is on average $15 \mathrm{~W} \mathrm{~m}^{-2}$ greater than CERES OLR. Incompletely offsetting this, CombRet net SW TOA radiation (i.e., TOA solar absorption) is $12 \mathrm{~W} \mathrm{~m}^{-2}$ larger than that of CERES; CombRet upwelling TOA SW flux 
TABLE 2. Time-mean (standard deviations) upwelling $(\uparrow)$ and downwelling $(\downarrow)$, longwave $\left(F_{\mathrm{LW}}\right)$ and shortwave $\left(F_{\text {Sw }}\right)$ radiative flux densities at the TOA and the surface (surf), and atmospheric flux convergences $\left(F_{\mathrm{atm}}\right)$, in $\mathrm{W} \mathrm{m}^{-2}$ for computed CERES Ed4 and CombRet. Negative convergences imply a cooling effect on the atmospheric column. Standard deviations are calculated using daily average values. All of the differences in mean values are statistically significant based on a paired Student's $t$ test at the $95 \%$ level. Correlations are calculated using daily mean values.

\begin{tabular}{|c|c|c|c|c|c|c|}
\hline & \multicolumn{3}{|c|}{ All sky } & \multicolumn{3}{|c|}{ Clear sky } \\
\hline & CERES & CombRet & Correlation & CERES & CombRet & Correlation \\
\hline$F_{\mathrm{LW}} \uparrow(\mathrm{TOA})$ & 237 (37) & $252(34)$ & 0.93 & $275(8)$ & $281(8)$ & 0.97 \\
\hline$F_{\mathrm{LW}} \uparrow$ (surf) & $472(3)$ & $471(3)$ & 0.86 & $471(3)$ & $471(3)$ & 0.84 \\
\hline$F_{\mathrm{LW}} \downarrow$ (surf) & $417(9)$ & $420(12)$ & 0.91 & 407 (6) & $410(7)$ & 0.88 \\
\hline$F_{\mathrm{SW}} \uparrow(\mathrm{TOA})$ & $96(50)$ & $87(54)$ & 0.85 & $40(3)$ & $36.4(0.3)$ & 0.85 \\
\hline$F_{\mathrm{SW}} \downarrow(\mathrm{TOA})$ & $420(7)$ & $423(7)$ & 0.98 & $420(7)$ & $423(6.5)$ & 0.98 \\
\hline$F_{\mathrm{SW}} \uparrow($ surf $)$ & $12.0(2.3)$ & $12(3)$ & 0.81 & $14.1(0.4)$ & $15.3(0.3)$ & 0.81 \\
\hline$F_{\text {SW }} \downarrow$ (surf $)$ & $237(52)$ & $248(65)$ & 0.84 & $295(8)$ & 307 (6) & 0.84 \\
\hline$F_{\mathrm{LW}, \mathrm{atm}}$ & $-182(32)$ & $-201(27)$ & 0.90 & $-211(6)$ & $-221(7)$ & 0.84 \\
\hline$F_{\mathrm{SW}, \mathrm{atm}}$ & $99(3)$ & $100(10)$ & 0.43 & $99(3)$ & $95(4)$ & 0.74 \\
\hline
\end{tabular}

is $9 \mathrm{~W} \mathrm{~m}^{-2}$ less, while CombRet downwelling TOA SW radiation is $3 \mathrm{~W} \mathrm{~m}^{-2}$ larger. The resulting net ( $\mathrm{SW}$ plus $\mathrm{LW}$ ) CombRet TOA heating is $3 \mathrm{~W} \mathrm{~m}^{-2}$ less than the CERES net TOA heating.

The slightly larger CombRet downwelling SW radiation is due to the different latitudinal extents of CombRet and CERES. Using CERES grid cells centered on $-1^{\circ} \mathrm{N}\left(-3^{\circ}\right.$ to $\left.1^{\circ} \mathrm{N}, 72^{\circ}-75^{\circ} \mathrm{E}\right)$, rather than on the equator, increases the CERES downwelling TOA SW flux by $3.8 \mathrm{~W} \mathrm{~m}^{-2}$, since the observational period corresponds to austral summer. Using a downwelling solar equal to that of CombRet would increase CERES solar fluxes by less than 1\%. Adjusting the CERES downwelling TOA SW radiation to agree with CombRet, assuming albedo is unchanged, reduces the compensation between the OLR and TOA SW differences, increasing the net TOA heating discrepancy to $5 \mathrm{~W} \mathrm{~m}^{-2}$.

Over the observational period, CombRet OLR is consistently larger than the corresponding CERES Ed4 OLR. Figure 3a compares CombRet to coincident CERES Ed4 3-hourly averaged OLR (dots). The large scatter is not surprising considering the narrow view of the Gan ARM site observations used in the CombRet retrieval, compared to the wide spatial average (over more than $10^{5} \mathrm{~km}^{2}$ ) of the satellite retrievals used in the CERES product. The larger symbols represent the data averaged into 10 bins, each with 96 realizations of 3-h means. The 3-h means are ordered and binned by three OLR products: by CombRet OLR (open blue circles), by CERES Ed4 OLR (open red circles), and by CERES Ed 3 OLR $($ red $\times$ s). Comparing the red $\times$ s to the red circles illustrates the difference between CERES Ed3 and Ed4. The distances from the red open circles (Ed4) to the one-to-one line are smaller than those from the red $\times \mathrm{s}(\mathrm{Ed} 3)$, and the Ed4 OLR bins are shifted to the right, especially for lower values of OLR, indicating higher mean OLR for Ed4, in closer agreement with CombRet.

Because the distribution of OLR is negatively skewed, with a mode at clear skies and a long tail of lower OLR, the spatial averaging of the CERES data flattens the distribution toward more intermediate values (Fig. 3b). Even taking this into account, CERES is systematically shifted toward lower OLR than CombRet.

CombRet OLR is $6 \mathrm{~W} \mathrm{~m}^{-2}$ greater than CERES Ed4 OLR for the highest values of OLR (Fig. 3a), suggesting a discrepancy in radiative fluxes even when clouds are almost absent. A similar offset in clear-sky OLR is present across all OLR values (reflected in the mean of Table 2), indicating a difference in mean atmospheric state independent of cloud fraction. Thus, some of the (all sky) OLR offset can be explained by differences in the mean profiles of water vapor or temperature used in the CERES and CombRet retrievals. Other clear-sky fluxes also exhibit statistically significant differences in their means (Table 2). For example, downwelling surface clear-sky LW radiation for CombRet is $3 \mathrm{~W} \mathrm{~m}^{-2}$ larger than for CERES. Nevertheless, the clear-sky difference in OLR is only $40 \%$ of the all-sky OLR difference, pointing to cloud properties as the dominant reason for the OLR difference between the two datasets.

Net (LW + SW) atmospheric radiative cooling is larger for CombRet compared to CERES Ed4 $\left(F_{\text {atm }}\right.$, Table 2), in agreement with Johnson et al. (2015) and Ciesielski et al.'s (2017) comparison of CombRet and CERES Ed3 (see Fig. 11 and Fig. 3, respectively). We obtain a CERES-to-CombRet atmospheric heating rate ratio of 0.83 using CERES Ed4 and 0.71 using CERES Ed3, straddling the Ciesielski et al. (2017) ratio of 0.76 . Ciesielski et al. (2017) calculate atmospheric heating between the surface and $70 \mathrm{hPa}$ for $(\sim 5)$ CERES grids 

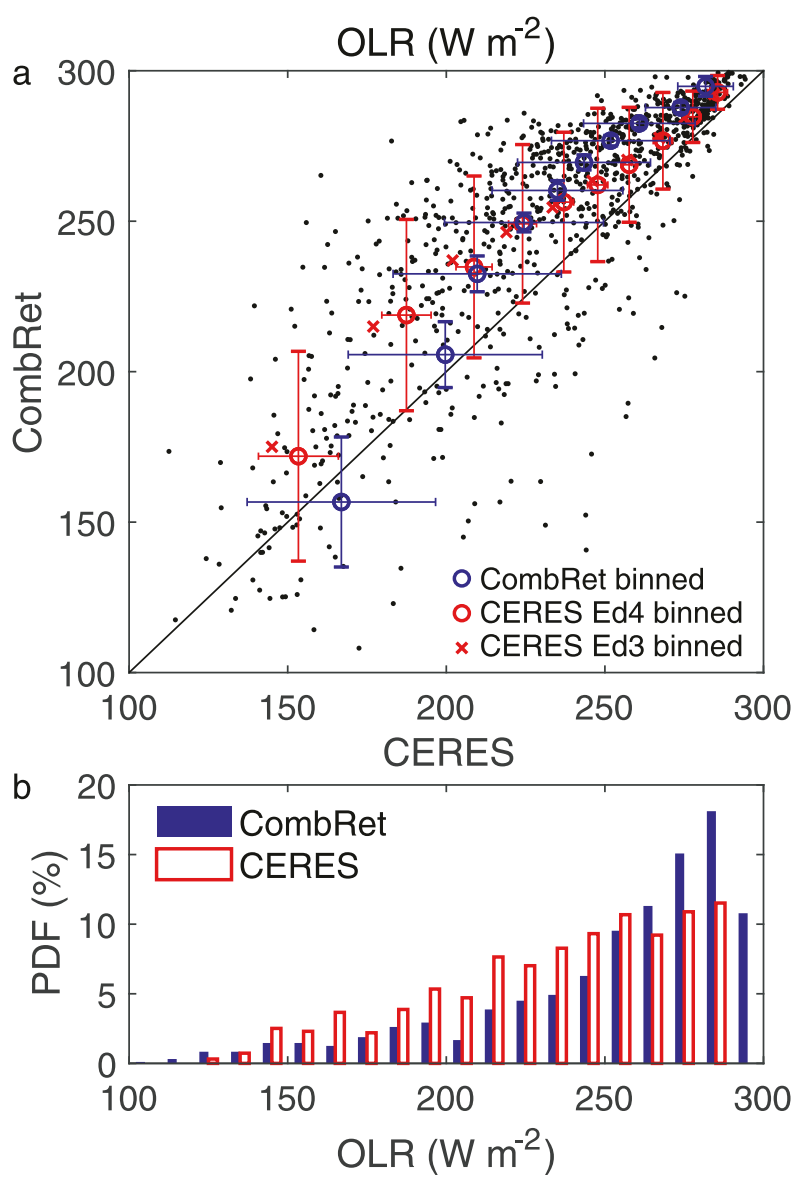

FIG. 3. (a) 3-hourly CombRet OLR from ground-based remote sensing vs coincident CERES Ed4 satellite-derived OLR (dots). The solid black line is the one-to-one line. Open blue circles represent the data averaged into 10 bins (of 96 realizations each), ordered by increasing CombRet OLR values, with the whiskers indicating standard deviations for each bin. Open red circles indicate binned averages based on increasing CERES Ed4 OLR, while red $\times s$ (without whiskers for clarity) show bins based on increasing CERES Ed3 OLR. (b) Probability distribution functions of CombRet and CERES Ed4 OLR.

within $150 \mathrm{~km}$ of Gan, while we calculate it for the entire column and our $3^{\circ} \times 4^{\circ}$ rectangle.

While SW fluxes differ little $\left(\leq 1 \mathrm{~W} \mathrm{~m}^{-2}\right)$ between CERES Ed 3 and Ed4, the Ed4 CERES dataset agrees better with CombRet LW fluxes. Compared with CERES Ed3, CombRet OLR is $21 \mathrm{~W} \mathrm{~m}^{-2}$ greater, and CombRet surface LW downwelling flux is $9 \mathrm{~W} \mathrm{~m}^{-2}$ greater, resulting in $30 \mathrm{~W} \mathrm{~m}^{-2}$ additional atmospheric cooling in CombRet (not shown). In comparison to CERES Ed4, CombRet OLR and surface downwelling LW fluxes are only 15 and $3 \mathrm{~W} \mathrm{~m}^{-2}$ greater, respectively, resulting in only $19 \mathrm{~W} \mathrm{~m}^{-2}$ additional atmospheric cooling in CombRet (Table 2). The clear-sky LW atmospheric cooling discrepancy between CombRet and CERES is reduced by roughly $25 \%$ when using CERES
Ed4 rather than Ed3, due to better agreement of Ed4 with the CombRet downwelling clear-sky LW surface flux. Although the all-sky and clear-sky flux differences are reduced, CombRet's atmosphere still emits more LW radiation both up and down, compared to CERES Ed4.

\section{b. Scenes responsible for the OLR difference}

Previous studies (e.g., Protat et al. 2014) indicate that the heights of the tops of the deepest clouds are underestimated by ground-based remote sensing when the remote sensing wavelengths are attenuated by thick cumulus clouds and rain. The CombRet retrieval partially reduces this bias by replacing deep precipitating cloud profiles from the shorter-wavelength Ka-band $(8.6 \mathrm{~mm})$ radar data with those from the longer-wavelength S-band $(10 \mathrm{~cm})$ radar data, which are much less affected by attenuation in precipitating clouds (Feng et al. 2014). Yet the retrieval does not compensate for this bias completely. Johnson et al. (2015) and Ciesielski et al. (2017) attribute the enhanced CombRet OLR, compared with CERES, to undersampling of high cloud due to attenuation of radar and lidar signals at the AMIE Gan site, especially total blockage of the lidar, but also note sources of errors in the CERES Ed3 data. For example, thin high-altitude clouds are likely underestimated by CERES (Del Genio and Chen 2015), and biases in the atmospheric state (temperature, water vapor, aerosols, etc.) used in the CERES radiative transfer calculations will be reflected in CERES computed fluxes. While CERES Ed4 improves on CERES Ed3, the gridded (level 3) products such as SYN1deg are still subject to errors and uncertainties (Loeb et al. 2018). The magnitude of improvement between these sequential versions of CERES, $6 \mathrm{~W} \mathrm{~m}^{-2}$ for computed OLR, serves as an estimate of CERES uncertainty. Furthermore, mean observed CERES OLR is $239,2 \mathrm{~W} \mathrm{~m}^{-2}$ larger than mean computed CERES OLR, providing an indicator of the error of the TOA fluxes (CERES_SYN1deg_Ed4A Data Quality Summary, available online at https:// ceres.larc.nasa.gov/documents/DQ_summaries/CERES_ EBAF_Ed4.0_DQS.pdf), in line with the mean monthly regional observed LW flux uncertainty of $2 \mathrm{Wm}^{-2}$ (CERES Terra/Aqua Edition4A SYN1deg TOA Flux Accuracy and Validation, available online at https:// ceres-tool.larc.nasa.gov/ord-tool/user/DQS/CERES_ SYN1deg_TOA_Ed4A.pdf). None of these CERES uncertainty estimates are as large as the $15 \mathrm{Wm}^{-2}$ difference between CERES Ed4 and CombRet OLR, so we conclude that much of this OLR discrepancy is due to bias in the CombRet product.

Figure $3 \mathrm{a}$ indicates that low-OLR scenes, characteristic of widespread high cloud (i.e., anvil and cirrus), 


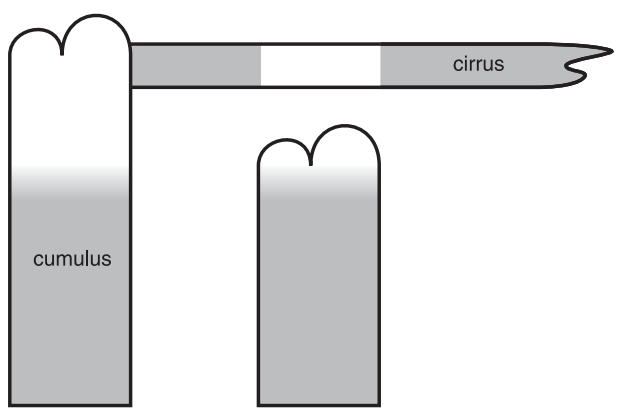

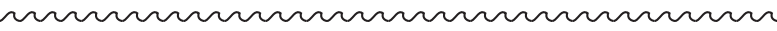

FIG. 4. Schematic illustrating underestimate of cloud top when deep cumulus are present. Gray fill indicates detection of cloud by ground-based lidar and radar, while white fill indicates signal attenuation. Cirrus clouds are sufficiently thin that ground-based remote sensing can accurately determine the cloud top.

show no consistent difference between between CERES and CombRet. On the other hand, high-OLR scenes, which are nearly clear, tend toward the clear-sky (cloudremoved) OLR difference (CombRet minus CERES) of $6 \mathrm{~W} \mathrm{~m}^{-2}$ (Table 2), again suggesting a mean difference in the noncloud atmospheric properties. Yet, the largest consistent overestimation of CombRet OLR relative to CERES occurs for scenes with intermediate OLR (roughly $220-260 \mathrm{~W} \mathrm{~m}^{-2}$ ). While widespread coverage of cirrus anvil clouds near the tropopause is responsible for the lowest daily average OLR, it does not correspond to the largest discrepancy between CERES and CombRet OLR.

We hypothesize that convectively precipitating, horizontally narrow, and vertically and optically thick towering cumulus clouds are responsible for many of these mid-OLR scenes. We further speculate that these clouds are responsible for the greatest attenuation of the ground-based radar and lidar used to detect cloud tops. In contrast to the towering convective cumulus clouds that detrain them, cirrus anvil clouds are optically thinner and precipitate only lighter stratiform rain. For this reason, we expect remote sensing wavelengths are less attenuated below high cirrus anvils, and thus cirrus anvils are less prone to underestimation from groundbased remote sensing than are cumulus clouds. Figure 4 schematically illustrates the underestimation of cloudtop height by CombRet in the presence of thick clouds.

In Fig. 3a, we examine the difference between CombRet and CERES OLR as a function of CERES OLR. Figure $5 b$ shows the difference between CombRet and CERES OLR as a function of rain rate from the S-Pol radar sited on Addu Atoll (Feng et al. 2014). The yellow line connects median differences for bins of sorted S-Pol rain values. Figure 5a, in turn, relates the S-Pol rain rate to CERES OLR. Here the yellow line

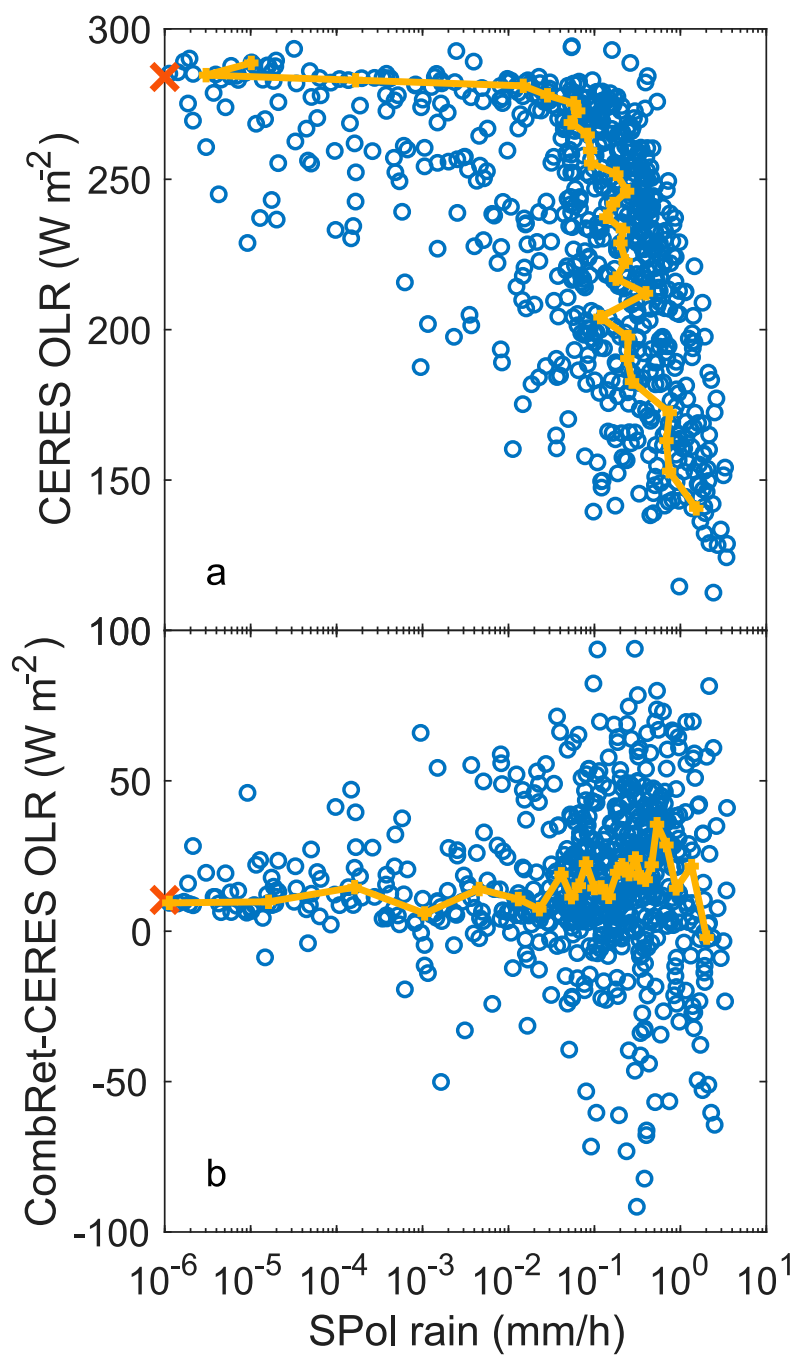

FIG. 5. (a) S-Pol rain rate (obtained from the DYNAMO Legacy Data project: http://dynamo.ml-ext.ucar.edu/dynamo_legacy/) vs CERES OLR. The yellow line connects median rain rates for bins of sorted CERES OLR. (b) Difference between CombRet and CERES OLR, with the yellow line indicating the differences for bins of sorted S-Pol rain rate values. The red $\times$ s on the $y$ axes indicate the averages for all nonprecipitating scenes.

connects median rain rates for CERES OLR bins. Figure $5 \mathrm{~b}$ indicates an increase in the (positive) CombRet OLR bias for S-Pol rain rates of $0.02-2 \mathrm{~mm} \mathrm{~h}^{-1}$, while Fig. 5a indicates that this range of rain rates is responsible for most of the realizations of intermediate $\left(\sim 200 \mathrm{~W} \mathrm{~m}^{-2}\right.$ ) CERES OLR. The sign and magnitude of the CombRet biases in this range of rain rates matches those of the peak CombRet biases seen in Fig. 3a. This further supports our hypothesis that these mid-OLR scenes correspond to the greatest attenuation of the ground-based radar and lidar. Note that this does not imply that only rain itself is responsible for the CombRet bias, but rather that scenes 


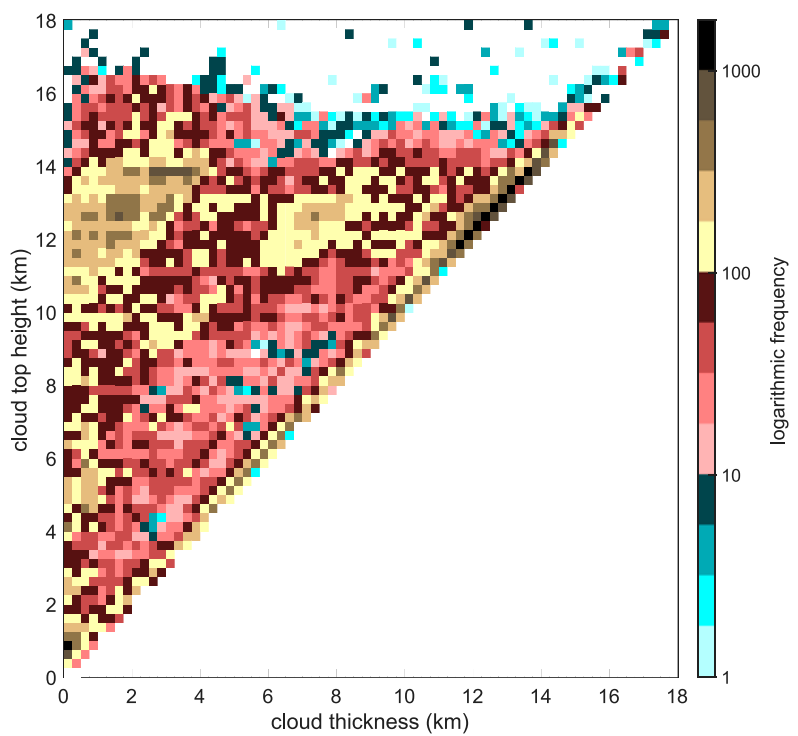

FIG. 6. Frequency of cloud-top height and cloud thickness values (counts in $0.25-\mathrm{km}$ cloud height/thickness bins), calculated from the CombRet 30-s resolution cloud mask.

with rain, and hence precipitating clouds, tend to contribute to the bias.

The increased OLR in CombRet compared to CERES suggests that CombRet has a sizable population of clouds with lower cloud-top heights (higher cloud-top pressures) compared to CERES. We cannot directly compare 3-hourly CERES and CombRet due to differences in sampling and averaging methodology. Thus, we explore the joint distribution of cloud-top height and cloud thickness in the high temporal (30 s) resolution CombRet data (Fig. 6), which precludes complications due to sampling and averaging. Many thin clouds have high cloud tops (height $>13 \mathrm{~km}$ ), while thicker clouds (thickness $>5 \mathrm{~km}$ ) are much less likely to have cloud tops this high. Thin high cirrus clouds consist of small ice crystals and persist a long time in the upper troposphere. When the size and concentration of crystals is too low, they are too thin to be detected by radar, and they are only detected by lidar when not blocked by intervening lower clouds. We speculate that some portion of the decrease in cloud-top height with increasing cloud thickness is due to an underestimation of the cloud-top heights in the presence of thicker cumulus clouds that block the lidar. This negative bias in cloud-top height would contribute to an overestimate of CombRet OLR (i.e., warmer clouds, emitting more LW radiation to space) compared to CERES.

\section{c. Isolating the effects of cloud-top height differences}

Figure 6 indicates that CombRet's thick clouds have lower cloud-top heights than thin clouds. While other

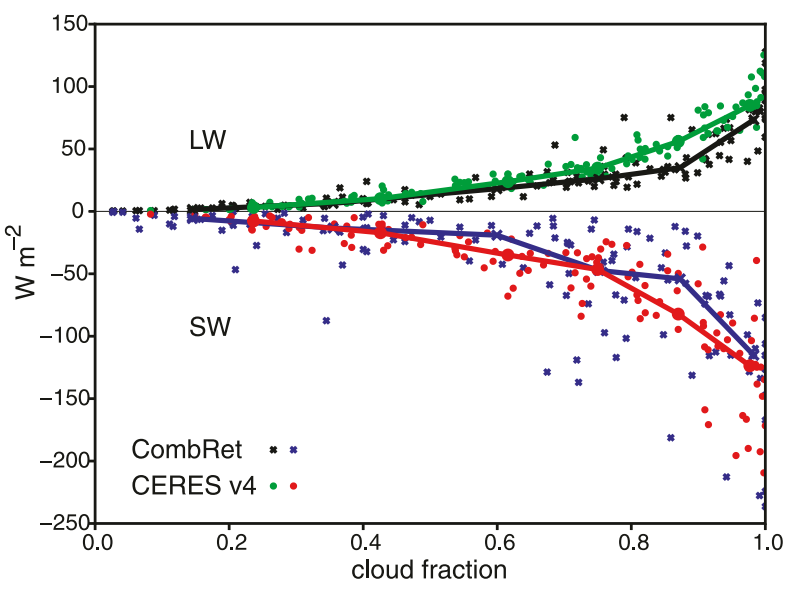

FIG. 7. Daily mean CombRet LW (small black $\times$ s), CERES Ed4 LW (small green circles), CombRet SW (small blue $\times$ s), and CERES Ed4 SW (small red circles) TOA cloud radiative effect as a function of the corresponding CombRet or CERES cloud fraction. The piecewise continuous lines connect medians (large symbols) in bins of 20 , sorted by the cloud fraction quantity. Positive values indicate clouds warm the column (surface plus atmosphere).

observations would be necessary to confirm that thick cloud-top heights are underestimated by CombRet, the decreased thick cloud height in Fig. 6 is consistent with an underestimate, as well as the higher OLR from CombRet compared with CERES. This is in line with what we expect would happen were the lidar beam obscured. However, this figure alone cannot provide the magnitude of the resulting OLR overestimate. Other cloud properties, specifically cloud fraction and cloud (geometric or optical) thickness, could also contribute to differences in OLR. Because 3-hourly cloud heights in CombRet and CERES are not comparable, we must examine other variables to investigate the relative importance of cloud fraction versus cloud height and thickness discrepancies. Figure 7 compares the daily mean CombRet $(\times s)$ and CERES (circles) TOA cloud radiative effect (CRE) and cloud fraction. We focus on CRE, rather than OLR, to reduce the effects of the known clear-sky differences. A positive CRE corresponds to a warming by clouds (due to either a decrease in OLR or a decrease in SW reflection).

Even for the same cloud fraction, CombRet TOA LW CRE (the decrease in OLR due to clouds; black $\times \mathrm{s}$ ) is lower (by about $20 \mathrm{~W} \mathrm{~m}^{-2}$ ) compared to CERES (green circles), especially in the 0.8 to 1.0 cloud fraction range. For cloud fraction less than 0.8, CombRet and CERES are in better agreement, but CombRet is still slightly lower. Surface LW CRE from CombRet is similar to that from CERES (not shown). These results are consistent with lower (warmer) tops for optically thick 
clouds in CombRet, as surface LW CRE is insensitive to cloud-top temperature.

The SW TOA CRE difference between CombRet (blue $\times$ s) and CERES (red circles) is similar, but with opposite sign and more scatter. For a cloud fraction between 0.5 and 0.9 , CombRet has more days with a relatively weak (negative) SW CRE, indicating lessreflective clouds. Since cloud temperature does not directly affect SW radiation, these weak-SW-CRE days likely result from optically thinner clouds. The timemean TOA albedo (time-mean reflected SW radiation divided by time-mean incoming SW radiation) for CombRet $(0.21)$ is a little lower than for CERES (0.23). This can be partially attributed to a slightly lower mean cloud fraction for CombRet (not shown), and CombRet also has a slightly lower clear-sky albedo (0.09 versus 0.10). Differences in the diurnal cycle of cloud fraction could also impact SW CRE. However, Fig. 7 indicates that, for the same cloud fraction, some CombRet clouds are less reflective than CERES clouds, which also contributes to the lower TOA albedo for CombRet. Surface SW CRE is weaker for CombRet, for a given cloud fraction, consistent with optically thinner clouds (not shown).

Thus, the differences between CERES and CombRet TOA fluxes cannot be solely attributed to the cloud fraction differences. Figure 7 indicates that, for high cloud fraction days, CombRet clouds have a tendency toward a smaller CRE. Lower (warmer) cloud tops reduce LW CRE, and optically thinner clouds reduce SW CRE. To further isolate the radiative effects of cloudtop height differences, we use a "cloud-layer albedo" metric, which responds to cloud fraction and optical depth, but not to height.

We estimate the cloud-layer albedo $\left(\alpha_{\mathrm{cld}}\right)$, the planetary albedo attributed to clouds, by modeling the SW budget as a single cloud layer above a surface layer. We neglect multiple reflection between the surface and cloud layer, justified by the low reflectivity of the surface. We use the clear-sky (cloud-removed) TOA albedo as the surface albedo $\left(\alpha_{\text {sfc }}\right.$, clear-sky reflected TOA SW divided by incoming TOA SW). In reality, the clear-sky albedo includes contributions from aerosols; however, high clouds are mostly above this aerosol layer, so we treat the aerosol reflectivity as part of the "surface" here. Thus, $\alpha_{\text {sfc }}$ could be called the "background" clear-sky reflection. The TOA planetary albedo $\left(\alpha_{\mathrm{TOA}}\right.$, all-sky reflected TOA SW divided by incoming TOA SW) is equal to $\alpha_{\text {cld }}+\left(1-\alpha_{\text {cld }}\right) \alpha_{\text {sfc. }}$. Rearranging this yields

$$
\alpha_{\mathrm{cld}}=\frac{\alpha_{\mathrm{TOA}}-\alpha_{\mathrm{sfc}}}{1-\alpha_{\mathrm{sfc}}} .
$$

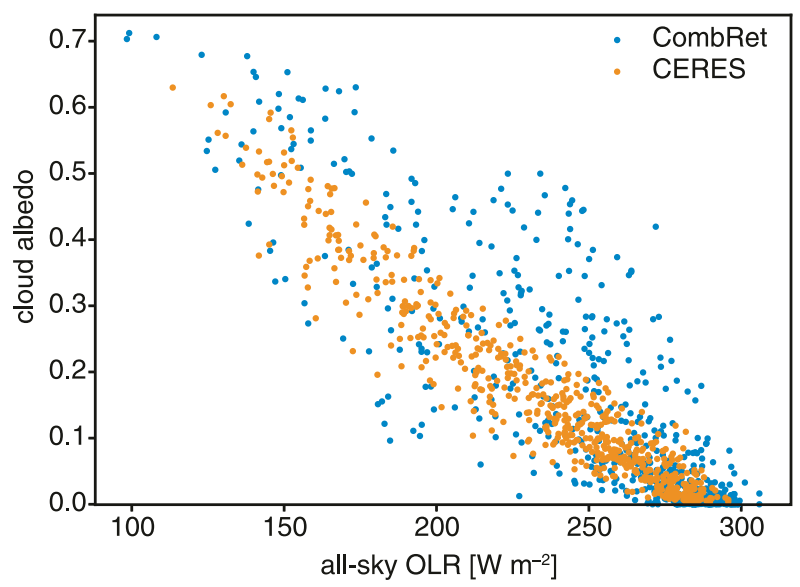

FIG. 8. 3-hourly (excluding nighttime) OLR and cloud-layer albedo for CombRet (blue dots) and CERES Ed4 (orange dots).

Using CERES and CombRet 3-hourly (excluding nighttime) TOA SW fluxes, we calculate $\alpha_{\mathrm{sfc}}$ and $\alpha_{\mathrm{TOA}}$ and estimate the cloud-layer albedo, shown in Fig. 8. The all-sky and clear-sky TOA albedo (not shown) both show a strong diurnal cycle, because of the specular reflectivity of the ocean surface. The cloudlayer albedo has the advantage of removing this dependence on solar angle. Thus $\alpha_{\text {cld }}$ is the cloud fraction weighted to give the SW reflection.

For CERES, this combined cloud fraction and thickness metric explains much of the OLR variation (orange dots in Fig. 8). Cloud-layer albedo decreases roughly linearly with OLR. CombRet (blue dots in Fig. 8) also shows a strong relationship between OLR and cloudlayer albedo. However, for a given cloud-layer albedo, CombRet sometimes has a higher OLR compared to CERES, especially for intermediate values of OLR.

Variations in cloud height will have little effect on the cloud albedo but a strong effect on OLR, resulting in no correlation between cloud albedo and OLR. On the other hand, variations in cloud fraction affect both cloud albedo and OLR. If cloud fraction increases while other cloud properties are held constant, cloud albedo will increase and OLR will decrease linearly. Low clouds increase the albedo, but have a small effect on OLR, whereas high clouds strongly affect both albedo and OLR. If variation in low cloud fraction were responsible for the variability in cloud albedo, we would expect only a small corresponding variation in OLR (i.e., an almost vertical linear relationship in Fig. 8). However, Fig. 8 shows that cloud-layer albedo decreases strongly with OLR, indicating that most of the observed temporal OLR variability is correlated with the fraction of high cold clouds, rather than cloud-top height, especially for CERES (orange dots). If CERES cloud-top height is varying, it is doing so in conjunction with cloud-layer 

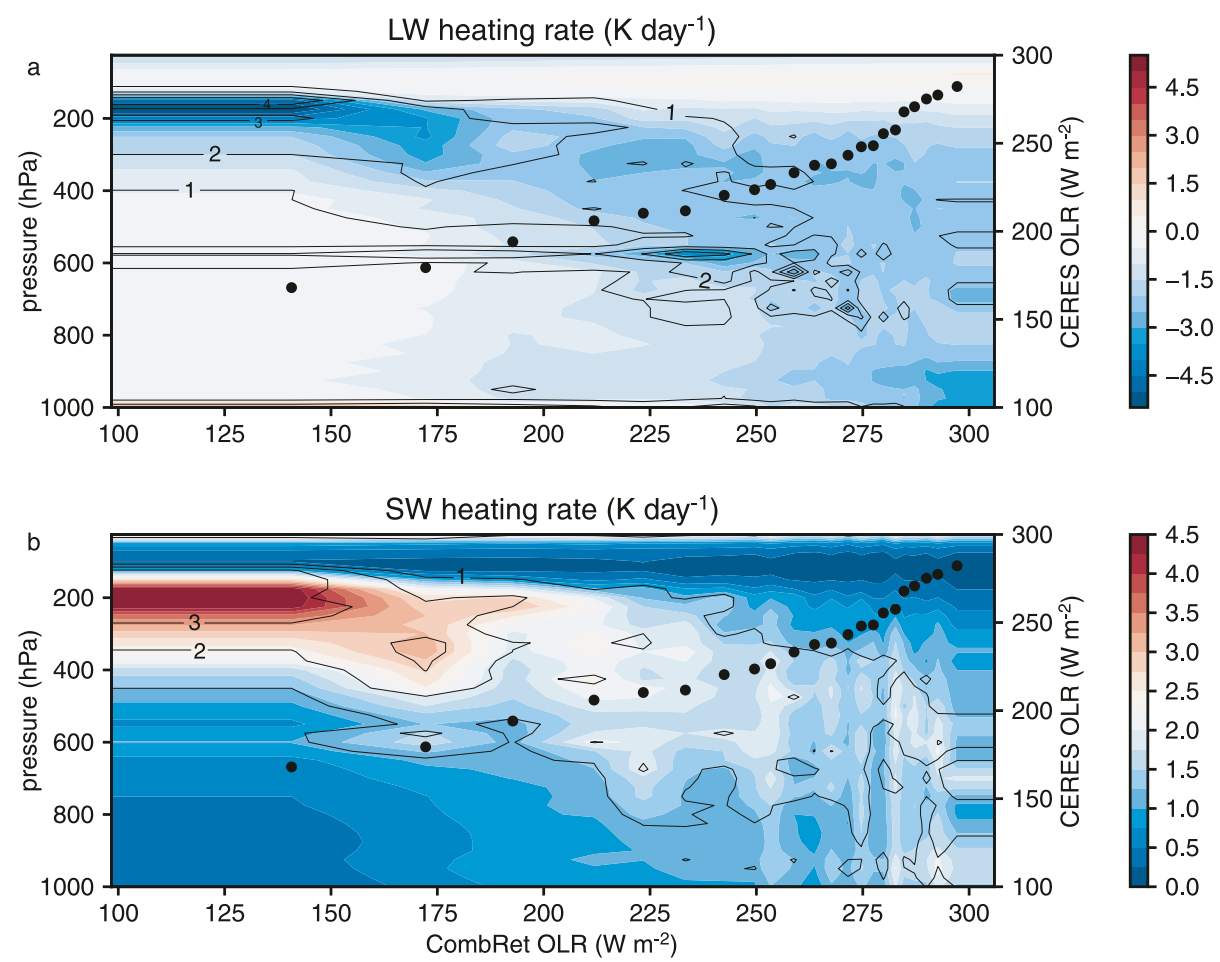

FIG. 9. Filled contours indicate Gan CombRet (a) LW and (b) SW heating rates $\left(\mathrm{K} \mathrm{day}^{-1}\right)$ sorted by CombRet OLR and composited by like OLR. Dots show the mean CombRet and CERES Ed4 OLR $\left(\mathrm{W} \mathrm{m}^{-2}\right.$ ) for each of the 22 bins. Each bin has 44 3-h realizations. Black contours (spaced by $1 \mathrm{~K} \mathrm{day}^{-1}$ ) indicate sample standard deviation within each heating rate composite. CombRet OLR is used for the horizontal axis, so the OLR bins are wider on the far left side of the figure, whereas the equally populated bins corresponding to high OLR are more finely resolved in OLR.

albedo. On the other hand, for CombRet, cloud-top height is behaving somewhat independently from cloudlayer albedo (blue dots). Especially for intermediate values of OLR, CombRet has higher cloud albedo than would be expected from the linear CERES relationship, consistent with the decreased LW CRE in Fig. 7 and lower (warmer) CombRet cloud tops in these cases. This supports our hypothesis that biases in the cloud-top height of CombRet thick clouds are responsible for much of the overestimate of OLR by CombRet.

\section{Composite CombRet vertical heating profiles}

TOA longwave radiation (i.e., OLR) is the most strongly varying component of the atmospheric column heat budget (Del Genio and Chen 2015). It varies intraseasonally mostly due to changes in cloud radiative effect. OLR is routinely measured by operational weather satellites, and it forms the basis for many schemes for identifying tropical convection, atmospheric waves, and intraseasonal variability.

Although there is uncertainty in the CERES product (section 4b), CERES instruments measure TOA radiation directly, compared to the CombRet surfacebased retrieval. CombRet underestimates cloud-top height, resulting in higher-than-observed intermediate and mean OLR in the CombRet data. Furthermore, the clear-sky OLR difference between CombRet and CERES is not correlated with (all-sky) OLR, suggesting differences in the vertical profiles of water vapor, temperature, and aerosols between the respective clear-sky atmospheres. Here, we combine the CERES and CombRet data to take advantage of the high vertical resolution and surface-based observations of CombRet as well as the more directly observed TOA fluxes from CERES. We composite the vertical profile of CombRet heating rates on CombRet OLR. From this we construct a vertically resolved time-height series consistent with both CombRet vertical profiles and CERES OLR fluxes.

First, we sort the CombRet 3-hourly LW and SW vertical heating rate profiles by CombRet OLR and then average profiles of like CombRet OLR in 22 bins, each with 44 3-h realization (filled, color contours in Fig. 9). This averaging simplifies the highly variable vertical structure of the heating rates. For the SW heating rate 

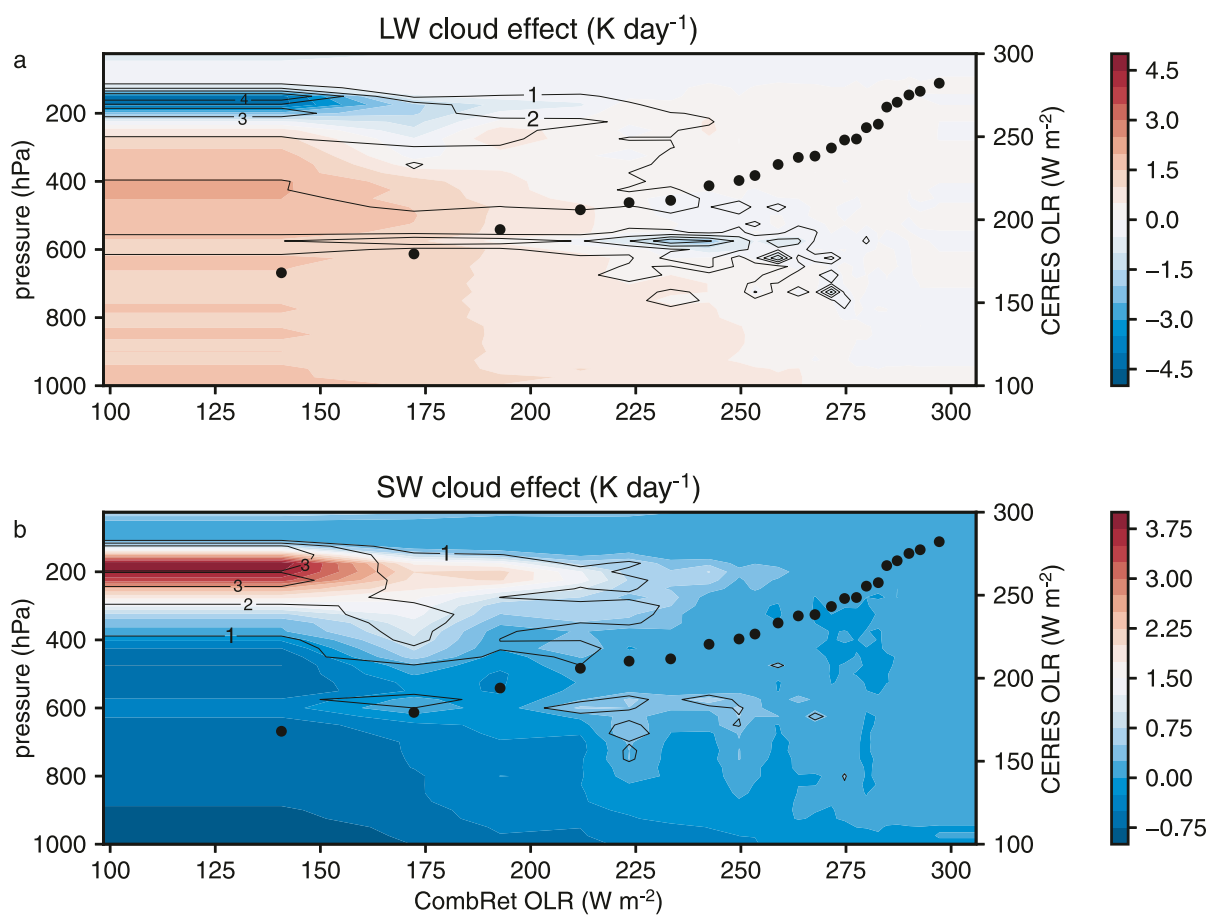

FIG. 10. As in Fig. 9, but for cloud radiative effect $\left(\mathrm{K} \mathrm{day}^{-1}\right)$.

profiles, composites are constructed using only daylight scenes. The results are not sensitive to the choice of bin number; sorting observations into 20 or 30 bins, rather than 22, produces similar results. We similarly construct OLR composites for cloud radiative effect (Fig. 10). Our radiative heating rate composites qualitatively corroborate radiative fields composited by low-pass filtered rain rates (Ciesielski et al. 2017, Fig. 6).

The sample standard deviations (among the 44 realizations for each OLR bin) in Figs. 9 and 10 (black contours) are nearly as large as the mean values for each bin. There is considerable variability in cloud properties not explained by OLR, because many different cloud configurations can result in the same OLR. There is a two-peaked vertical structure for the maximum standard deviation of LW cooling and SW warming at cloud top. This can be explained by vertical shifting of the distribution of the cloud top within the 3-h means that comprise the composites. Shifting the gradient of the radiative heating at the edge of the cloud-top distribution generates the most variance in the radiative effects.

While the sample standard deviations are of similar magnitudes to the mean heating rates, the $95 \%$ significance level of the mean is $0.30 \mathrm{Kday}^{-1}$ for LW and $0.37 \mathrm{~K} \mathrm{day}^{-1}$ for the SW composites. Thus, much of the structure of these mean heating rates is statistically significant. The radiative effects are coherent across several bins of OLR and pressure levels, so averaging independent adjacent bins together would increase statistical significance without changing the results. Since the heating rates and CREs converge to the OLR composite means, we expect them to be representative of variability over an intraseasonal cycle, over a month of data, or over spatial averages of regions with similar OLR. While there is certainly internal variability not explained by OLR, these composites are appropriate for characterizing heating rates among the phases of the MJO.

The composite LW cloud radiative effect steadily decreases as OLR increases (Fig. 10a). For OLR below $200 \mathrm{~W} \mathrm{~m}^{-2}$, LW CRE has a sharp minimum around $200 \mathrm{hPa}$. This composite represents the combined effects of optically thick clouds, which have enhanced cooling at cloud top and warming at cloud base, and thin cirrus clouds, which warm throughout the cloud. The enhanced cooling of thick clouds is greater than the warming due to thin clouds, resulting in the 200-hPa LW CRE minimum. Cloud SW absorption warms the upper atmosphere (Fig. 10b), compensating for about half of the LW CRE. In the lower troposphere (pressures $\geq$ $\sim 400 \mathrm{hPa}$ ), a reduction in downwelling SW radiation results in cooling CRE below clouds. These LW and SW CRE profiles indicate that persistent, widespread, and optically thick high clouds dominate the heating rate for OLR $<200 \mathrm{~W} \mathrm{~m}^{-2}$.

For OLR greater than $200 \mathrm{~W} \mathrm{~m}^{-2}$, a minimum LW CRE appears around $600 \mathrm{hPa}$, partly associated with the 

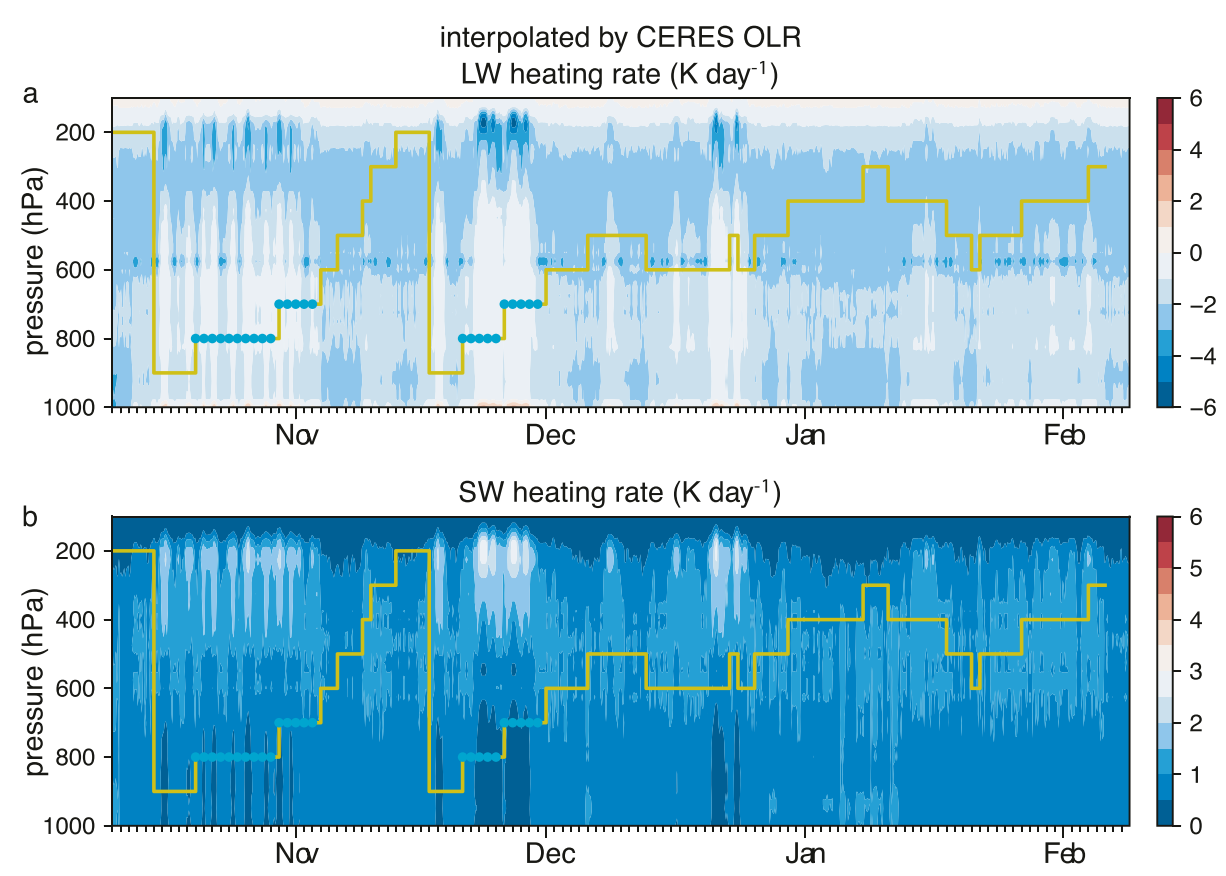

FIG. 11. Interpolated time-height series of CombRet (a) longwave and (b) shortwave atmospheric heating rates $\left(\mathrm{K} \mathrm{day}^{-1}\right)$, using the time series of CERES Ed4 observed OLR as the interpolant. In (b), the SW heating rate is shown only for daytime scenes (five per day), and the magnitudes are multiplied by $5 / 8$. The daily phase of the Real-time Multivariate MJO index (RMM; Wheeler and Hendon 2004) is indicated by the yellow lines; phases 2 and 3 (active phases) are highlighted with light blue circles. By construction, these interpolated heating rates correspond to OLR in agreement with CERES OLR, while the profiles themselves are consistent with the average Gan retrievals that match those CERES OLR values. Note that the color bar scale in (b) differs from that of Fig. 1b.

high emissivity retrieved by CombRet at the melting level ("bright band"). The melting level usually generates a SW warming artifact, but its effect on the LW heating rate retrieval is subtle: melting levels at cloud top generate a LW cooling artifact, but melting levels inside clouds often generate a LW warming artifact (cf. Fig. 14 of Feng et al. 2014). The higher radar reflectivity of the large liquid water drops relative to the ice particles aloft results in an artificial emissivity gradient that cools the melting level (by LW emission, similar to a cloud top) in the radiative transfer calculation. Our long-term composites have mean LW cooling at the melting level. Some of this may be due to a true mode of freezing-level cumulus cloud tops, but this altitude is at least somewhat contaminated by meltinglevel heating rate artifacts in the radar retrievals.

The so-called bright band cloud radiative effect on heating rate is largest for OLR of $220-260 \mathrm{~W} \mathrm{~m}^{-2}$, where we also see the largest discrepancy between CombRet and CERES OLR (Fig. 3a and section $4 \mathrm{~b}$ ). The binned LW CRE is negative where there is weak stratiform rain. The melting level has the highest reflectivity within the column and, hence, acts as a cloud top. These $600-\mathrm{hPa}$ bright band cloud tops, which are actually melting-band rain, are likely to result in scenes with higher OLR in CombRet.

These 22 composite heating rate profiles (binned by CombRet OLR, shown in Fig. 9 and Fig. 10) can be used as a look-up table to derive CombRet-consistent heating rates matching OLR retrievals from another source. As an example, in Fig. 11, we create time-height series of LW and SW heating rates, using the CERES observed OLR time series as the interpolant. First, for each CombRet radiative term $R(t)$ as a function of time $t$, we sort by corresponding OLR and average to get $R^{\prime}(\mathrm{OLR}) . R^{\prime}$ is linearly interpolated between adjacent CombRet-OLR bin means. We then evaluate this function for the 3-hourly CERES observed OLR values $\left(\mathrm{OLR}_{\mathrm{CERES}}\right)$ to get the adjusted time series, $R_{\text {adj }}(t)=R^{\prime}$ $\left[\operatorname{OLR}_{\text {ceres }}(t)\right]$. In other words, we select the location along the $x$ axis of Fig. 9 that corresponds to the CERES OLR and interpolate between the two bracketing CombRet OLR dots. For the SW heating rates, we exclude night and multiply by $5 / 8$ to account for the fraction of scenes at night when SW heating is zero. Since each OLR-binned heating rate is a 134-h mean, the new time series averages out individual mesoscale storms shown in Fig. 1. 

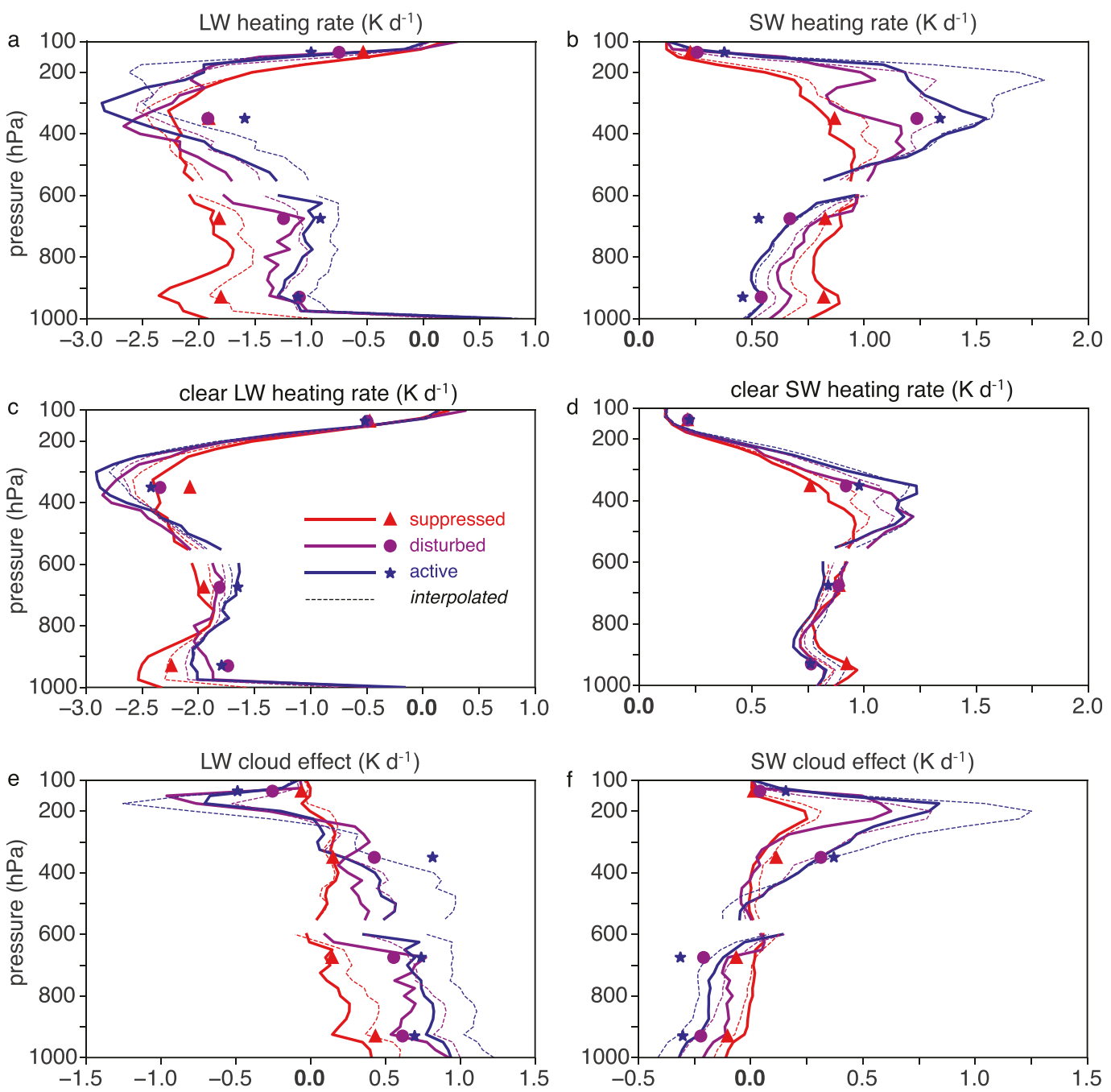

FIG. 12. Average heating rate profiles for the convectively suppressed (red; RMM phase 4-8), convectively disturbed (purple; phase 1), and convectively active (blue; phase 2-3) phases of the Real-time Multivariate MJO index. Solid curves show the original heating rates (i.e., averages of Fig. 1 data shown in Fig. 2). Dashed curves show heating rates adjusted to match CERES observed OLR (i.e., averages of Fig. 11). Symbols indicate CERES Ed4 computed heating rates at four pressure levels.

Figure 12 compares the resulting mean CERESinterpolated (dotted) convectively suppressed (red), disturbed (purple), and active (blue) phase heating rates with the original CombRet heating rates (solid). The distribution of CERES OLR is broadly shifted to lower OLR compared with CombRet (Fig. 3b). The interpolation using observed CERES OLR (mean OLR = $239 \mathrm{~W} \mathrm{~m}^{-2}$ ) chooses CombRet scenes that have on average $13 \mathrm{~W} \mathrm{~m}^{-2}$ lower OLR, which thus have systematically more and higher clouds than the contemporaneous CombRet scenes. Despite the fact that we do nothing to constrain the SW adjustment, the interpolation method produces reasonable SW heating rates.

The increased cloud amount in the CERES-interpolated profiles compared with the CombRet profiles can be clearly seen in the SW CRE (Fig. 12f), where all phases show a stronger positive CRE in the upper troposphere and a stronger negative CRE in the lower troposphere. The additional upper tropospheric cloud heating in the interpolation gets stronger and higher as the MJO phase transitions from suppressed to active, when there are more high clouds.

For the suppressed phase, the SW clear-sky heating rate also increases between roughly 300 and $500 \mathrm{hPa}$ (Fig. 12d), suggesting increased upper-tropospheric water vapor in the CERES-interpolated profiles, which is also consistent with the increased upper-troposphere LW clear-sky cooling (Fig. 12c) and the corresponding reduced SW and LW clear-sky heating rate magnitudes in the lower troposphere. The suppressed heating rate 
profiles are essentially moving closer to the original disturbed profiles (with increased high clouds and water vapor), in order to match the CERES OLR.

For the convectively active phase, CombRet LW cooling has a \pm 100 -hPa-wide peak centered at $300 \mathrm{hPa}$ (Fig. 12a, solid blue line). In contrast, the interpolated active-phase LW cooling (blue dotted line) is weaker but extends higher $\left(-2.5 \mathrm{~K} \mathrm{day}^{-1}\right.$ from 300 to $\left.200 \mathrm{hPa}\right)$. The CERES-interpolated LW heating rate has less cooling than CombRet, uniformly from $300 \mathrm{hPa}$ to the surface. The LW CRE (Fig. 12e) is amplified, with a stronger cooling effect above $200 \mathrm{hPa}$, and a stronger warming effect below $250 \mathrm{hPa}$, consistent with increased high clouds. The decrease in LW clear-sky cooling around $300-400 \mathrm{hPa}$ (Fig. 12c) suggests these profiles are either cooler or have less water vapor. The SW clear-sky heating does not change much (Fig. 12d). A cooler upper troposphere would lower OLR when clouds are present. Thus, there is the possibility that some of the decreased OLR in the CERES-interpolated dataset compared with CombRet could be due to a colder atmosphere, in addition to an increase in high clouds.

For the original (uninterpolated) CombRet data, the difference between the disturbed and active phases is not robust. The disturbed phase has few degrees of freedom, and the mean disturbed heating rate is often within the standard error of the mean active phase heating rate (Fig. 2). We therefore have little confidence in the changes between the original and interpolated disturbed heating rates (solid and dashed purple lines in Fig. 12, respectively). Qualitatively, as with the original data, the interpolated disturbed heating rate lies between the interpolated active and suppressed heating rates, and the disturbed phase (all sky) LW heating rates are mostly shifted more positive (Fig. 12a).

CERES provides modeled radiative fluxes at 850,700 , 500,200 , and $70 \mathrm{hPa}$, between which we compute radiative heating centered on $925,675,350$, and $135 \mathrm{hPa}$, for the suppressed, disturbed, and active phase of the RMM over Gan (triangle, circle, and star respectively in Fig. 12). The heating rates for the CERES layers mostly agree with the more highly resolved CombRet heating rate composites, falling within their sampling standard deviations (Fig. 2). CERES heating rate differences among the suppressed, disturbed, and active phases are also similar to those of the CombRet composites.

An exception, where the CERES and CombRet heating rates disagree, is that CERES has less LW cooling than CombRet between 200 and $500 \mathrm{hPa}$ (Fig. 12a). This disagreement is reduced for the CERES-interpolated profile. The CombRet LW cloud effect at this height (Fig. 12e) is weaker (less positive) than CERES. While the CombRet LW cloud effect transitions from cooling above $250 \mathrm{hPa}$ to warming below $350 \mathrm{hPa}$, the CERES LW cloud effect in the 350-hPa layer is similar to the LW cloud effect in lower layers, with stronger warming in the active phase $\left(+1 \mathrm{~W} \mathrm{~m}^{-2}\right.$ in the 350 -hPa layer) than in the suppressed phase. The CERES-interpolated LW CRE better matches this behavior at $350 \mathrm{hPa}$.

A limitation of the present implementation of our methodology is that it includes only changes that can be parameterized by the interpolant OLR, yet there are different ways to modify the atmospheric column to achieve a lower OLR: from increasing high clouds, to decreasing temperature, to increasing water vapor. Furthermore, the composites constructed from CombRet that do represent high clouds may be biased toward cases with reduced lowcloud fraction or thickness, because ground-based lidar and radar only sense the highest-topped clouds when their signal is not attenuated.

\section{Conclusions}

We use the PNNL CombRet surface and TOA radiative fluxes and vertical radiative heating rates to study MJO behavior at Gan Island, Maldives $\left(0.5^{\circ} \mathrm{N}\right.$, $73^{\circ} \mathrm{E}$ ), during the AMIE-Gan/DYNAMO campaign from October 2011 to February 2012. The PNNL CombRet product captures the behavior of two distinct MJO cycles. The MJO active phase is characterized by deep vertical convective structures, and followed by the suppressed phase, which has sparse low clouds and high OLR.

Mean heating rates for convectively suppressed (RMM 4-8), convectively disturbed (RMM 1), and convectively active (RMM 2-3) days indicate that column net radiative heating acts as a positive feedback to MSE anomalies, in agreement with previous work (Sobel et al. 2014; Johnson et al. 2015; Del Genio and Chen 2015; Ciesielski et al. 2017). The active phase's atmospheric radiative cooling is roughly half that of the suppressed phase, which would tend to amplify (the positive) active phase MSE anomalies. Both the LW and SW heating rates contribute to this warming anomaly (i.e., the active phase has both enhanced SW warming and decreased LW cooling), but the anomalous SW absorption contributes only about $20 \%$ of the net heating difference between the two phases.

We compute a measure of the contribution of radiation to the gross moist stability,

$$
\mathrm{GMS}_{\mathrm{rad}}=\frac{R}{P},
$$

as the ratio of the net radiative heat source ( $R$, which is negative) in the atmospheric column over the precipitation ( $P$, derived from the S-Pol rain rate). If both 
radiation and precipitation are in dynamic heating units $\left(\mathrm{W} \mathrm{m}^{-2}\right)$, then $\mathrm{GMS}_{\mathrm{rad}}$ is dimensionless. For the suppressed MJO phase, $\mathrm{GMS}_{\mathrm{rad}}$ is -0.93 using CombRet data, and slightly (10\%) less, -0.84 , using the adjusted radiative sources $R^{\prime}\left[\operatorname{OLR}_{\mathrm{CERES}}(t)\right]$. GMS $\mathrm{Gad}_{\mathrm{rad}}$ calculated using CombRet $R$ is -0.20 in the disturbed and -0.26 in the active phase, whereas $\mathrm{GMS}_{\text {rad }}$ calculated using $R^{\prime}$ $\left[\operatorname{OLR}_{\text {CERES }}(t)\right]$ is -0.16 in both disturbed and active phases, and thus less negative than CombRet by $20 \%-40 \%$.

CombRet provides high vertical and temporal resolution directly over Gan Island. However, the groundbased remote sensing it uses may underestimate high clouds, due to attenuation of the remote sensing wavelengths. To address this and test the validity of the CombRet merged dataset, we compare the time series of 3-hourly CombRet TOA and surface radiative fluxes with collocated LW and SW, and all-sky and clear-sky fluxes from the CERES SYN1deg-3Hour Ed4A product. All TOA and surface fluxes are correlated in time between the two datasets (correlation coefficients $\geq 0.81$ ). The agreement indicates that the heating directly over Gan Island is representative of the larger-scale behavior on the $4^{\circ}$ latitude by $3^{\circ}$ longitude CERES grid.

While they are correlated in time, some of the mean fluxes differ between CERES Ed4 and CombRet. CombRet estimates greater OLR (by $15 \mathrm{~W} \mathrm{~m}^{-2}$ ) and TOA net SW (downwelling minus reflected) heating (by $12 \mathrm{~W} \mathrm{~m}^{-2}$ ) than CERES. Net (SW + LW) CombRet atmospheric radiative cooling is $19 \mathrm{~W} \mathrm{~m}^{-2}$ smaller compared to CERES. CombRet's atmosphere emits more $\mathrm{LW}$ radiation both up and down, compared to CERES Ed4.

Previous work (Johnson et al. 2015; Ciesielski et al. 2017) used CERES Ed3, an older version of the CERES product. Assuming improvements in the Ed4 product (Doelling et al. 2018), we compare both CERES Ed3 and Ed4 to CombRet. The discrepancy between CombRet and CERES atmospheric LW radiative cooling is reduced by more than a third by using CERES Ed4 rather than Ed3, and the clear-sky atmospheric LW discrepancy is reduced by $25 \%$.

Johnson et al. (2015) and Ciesielski et al. (2017) suggest that CombRet high clouds are undersampled, contributing to the positive bias in CombRet OLR. To investigate the reason for the difference between CERES and CombRet, we compare 3-hourly OLR for CombRet and CERES Ed4. Scenes characteristic of wide-spread high cloud (i.e., lowest OLR) show no consistent difference between between CERES and CombRet. Scenes that are essentially clear, and have highest OLR, tend toward the clear-sky (cloud-removed) OLR difference (CombRet minus CERES) of $6 \mathrm{~W} \mathrm{~m}^{-2}$, indicating that some part of the OLR bias results from differences in atmospheric temperature or water vapor profiles. Nevertheless, the largest consistent overestimation of CombRet OLR relative to CERES occurs for scenes with intermediate values of OLR (roughly $220-260 \mathrm{~W} \mathrm{~m}^{-2}$ ) and rain rates of $0.02-2 \mathrm{~mm} \mathrm{~h}^{-1}$.

We hypothesize that these scenes correspond to convectively precipitating, horizontally narrow, and vertically and optically thick towering cumulus clouds. Thick and raining convective cumulus clouds attenuate more ground-based lidar and radar than thin cirrus do, leading to an undermeasurement of cumulus cloud-top height in the CombRet retrieval, and an overestimate of OLR when thick cumulus clouds are present. We also note that, when binned by OLR, CombRet vertical heating rate profiles show the largest "bright band" effects in this same OLR range, suggesting that melting-level artifacts may be contributing to this OLR overestimate.

A reduced cloud-top height is not the only cloud property that could lead to biased OLR. A decrease in cloud fraction (where clouds have the same cloud-top height, but are present less often) would also result in an overestimate of OLR. Similarly, optically thinner clouds would increase OLR. However, comparison of CERES and CombRet cloud radiative effects (CRE) indicates that the CRE differences are not solely due to cloud fraction differences. CERES clouds are more effective at reducing OLR for a given cloud fraction. Even if the cloud fractions were the same, CERES OLR would be smaller than CombRet OLR. Additionally, CERES OLR shows a tighter relationship with cloud-layer albedo, while a number of intermediate-OLR CombRet scenes have higher OLR than expected given the cloudlayer albedo. These results, while not providing a direct attribution of the CombRet OLR bias, support our hypothesis that biases in the cloud-top height of CombRet thick clouds substantially contribute to the overestimate of OLR by CombRet.

Unfortunately, this cloud-top height bias in CombRet limits the use of CombRet data for some applications. Johnson et al. (2015) adjust the profile of radiative heating by a constant fraction at all vertical levels to match the vertical integral from the collocated CERES product. The CERES product provides a more trustworthy estimate of OLR, but this adjustment does not take into account the vertical structure of the CombRet bias.

We develop an empirical method for parameterizing solar and longwave atmospheric heating profiles as a function of OLR. We resample statistically significant averages of CombRet heating rates to create a new time series that matches satellite-observed (CERES Ed4) OLR. First, we composite 3-hourly CombRet vertical 
LW and SW heating profiles by their OLR to create 22 "OLR-composited" profiles. These composites are appropriate for characterizing heating rates within the MJO. From the OLR-composited profiles we interpolate a time series of atmospheric radiative heating rate profiles, using CERES Ed4 observed OLR as the interpolant.

The resampled radiative heating rates emphasize the radiative effect of high clouds, especially in the convective phase of the MJO. The mean clear-sky heating rate profiles suggest changes in temperature and/or water vapor (or absorbing aerosol) in the OLRinterpolated atmosphere, compared to the CombRet atmosphere. Since this methodology matches observed OLR, not clear-sky OLR, changes are not constrained to clouds; however, Fig. 8 indicates that cloud radiative effects are responsible for most of the variability in OLR.

This strategy of using available high vertical resolution profiles with accurate and available satellite OLR, applied here to CombRet and CERES, could be applied to other types of variability and datasets. The method is accurate provided that the composites are constructed from samples that sufficiently represent the (temporal) variability of interest, and provided that the variability of the radiatively important parameters can be parameterized accurately by OLR.

A general benefit of the "OLR-composited" methodology is that it simplifies the comparison of cloud radiative effects between satellite products and modeled data. Clear-sky fluxes are calculated using the "cloudremoved" methodology of CombRet, rather than the "cloud-free-pixel" methodology of many satellite products. Thus, we can use satellite-observed all-sky flux (e.g., OLR), which is independent of the cloudremoved sampling, as the interpolant to generate a vertical CRE profile that is appropriate for comparison with model data.

Acknowledgments. This study was undertaken with support from NOAA CVP Grants NA13OAR4310160 and NA15OAR4310240, and Office of Naval Research Grant N00014-16-1-3094. Dr. Feng was supported by the Atmospheric Science Research (ASR) program as part of the DOE Office of Biological and Environmental Research. We acknowledge Paul Ciesielski, discussions with whom informed this work, as well as three anonymous reviewers. The AMIE/DYNAMO CombRet data were obtained from the U.S. Department of Energy Atmospheric Radiation Measurement data archive (ARM; https://doi.org/10.5439/1169498). CERES data were obtained from the Atmospheric Science Data Center at the NASA Langley Research Center (https://cerestool.larc.nasa.gov/ord-tool/jsp/SYN1degEd4Selection.jsp;
https://doi.org/10.5067/Terra + Aqua/CERES/SYN1deg3Hour_L3.004A). The S-Pol rain rate data were obtained from the DYNAMO Legacy Data project (http:// dynamo.ml-ext.ucar.edu/dynamo_legacy/).

\section{REFERENCES}

Andersen, J. A., and Z. Kuang, 2011: Moist static energy budget of MJO-like disturbances in the atmosphere of a zonally symmetric aquaplanet. J. Climate, 25, 2782-2804, https://doi.org/ 10.1175/JCLI-D-11-00168.1.

Ciesielski, P. E., R. H. Johnson, X. Jiang, Y. Zhang, and S. Xie, 2017: Relationships between radiation, clouds, and convection during DYNAMO. J. Geophys. Res. Atmos., 122, 2529-2548, https://doi.org/10.1002/2016JD025965.

Comstock, J. M., A. Protat, S. A. McFarlane, J. Delanoë, and M. Deng, 2013: Assessment of uncertainty in cloud radiative effects and heating rates through retrieval algorithm differences: Analysis using 3 years of ARM data at Darwin, Australia. J. Geophys. Res. Atmos., 118, 4549-4571, https:// doi.org/10.1002/JGRD.50404.

Del Genio, A. D., and Y. Chen, 2015: Cloud-radiative driving of the Madden-Julian oscillation as seen by the A-Train. J. Geophys. Res. Atmos., 120, 5344-5356, https://doi.org/ 10.1002/2015JD023278.

Doelling, D., C. Haney, R. Bhatt, B. Scarino, and A. Gopalan, 2018: Geostationary visible imager calibration for the CERES SYN1deg edition 4 product. Remote Sens., 10, 288, https:// doi.org/10.3390/rs10020288.

Feng, Z., S. A. McFarlane, C. Schumacher, S. Ellis, J. Comstock, and N. Bharadwaj, 2014: Constructing a merged cloudprecipitation radar dataset for tropical convective clouds during the DYNAMO/AMIE experiment at Addu Atoll. J. Atmos. Oceanic Technol., 31, 1021-1042, https://doi.org/ 10.1175/JTECH-D-13-00132.1.

Inoue, K., and L. E. Back, 2015: Gross moist stability assessment during TOGA COARE: Various interpretations of gross moist stability. J. Atmos. Sci., 72, 4148-4166, https://doi.org/ 10.1175/JAS-D-15-0092.1.

Johnson, R. H., P. E. Ciesielski, J. H. Ruppert, and M. Katsumata, 2015: Sounding-based thermodynamic budgets for DYNAMO. J. Atmos. Sci., 72, 598-622, https://doi.org/10.1175/JAS-D14-0202.1.

Kato, S., N. G. Loeb, F. G. Rose, D. R. Doelling, D. A. Rutan, T. E. Caldwell, L. Yu, and R. A. Weller, 2013: Surface irradiances consistent with CERES-derived top-of-atmosphere shortwave and longwave irradiances. J. Climate, 26, 2719-2740, https:// doi.org/10.1175/JCLI-D-12-00436.1.

Lin, J.-L., and B. E. Mapes, 2004: Radiation budget of the tropical intraseasonal oscillation. J. Atmos. Sci., 61, 2050-2062, https:// doi.org/10.1175/1520-0469(2004)061<2050:RBOTTI>2.0.CO;2.

Loeb, N. G., and Coauthors, 2018: Clouds and the Earth's Radiant Energy System (CERES) Energy Balanced and Filled (EBAF) top-of-atmosphere (TOA) Edition 4.0 data product. J. Climate, 31, 895-918, https://doi.org/10.1175/ JCLI-D-17-0208.1.

Ma, D., and Z. Kuang, 2011: Modulation of radiative heating by the Madden-Julian Oscillation and convectively coupled Kelvin waves as observed by CloudSat. Geophys. Res. Lett., 38, L21813, https://doi.org/10.1029/2011GL049734.

Madden, R. A., and P. R. Julian, 1971: Detection of a 40-50 day oscillation in the zonal wind in the tropical Pacific. J. Atmos. 
Sci., 28, 702-708, https://doi.org/10.1175/1520-0469(1971) 028<0702:DOADOI $>2.0$. CO;2.

Minnis, P., and Coauthors, 2011a: CERES Edition-2 cloud property retrievals using TRMM VIRS and Terra and Aqua MODIS data-Part I: Algorithms. IEEE Trans. Geosci. Remote Sens., 49, 4374-4400, https://doi.org/10.1109/ TGRS.2011.2144601.

, and Coauthors, 2011b: CERES Edition-2 cloud property retrievals using TRMM VIRS and Terra and Aqua MODIS data-Part II: Examples of average results and comparisons with other data. IEEE Trans. Geosci. Remote Sens., 49, 44014430, https://doi.org/10.1109/TGRS.2011.2144602.

Neelin, J. D., and I. M. Held, 1987: Modeling tropical convergence based on the moist static energy budget. Mon. Wea. Rev., 115, 3-12, https://doi.org/10.1175/1520-0493(1987)115<0003: MTCBOT $>2.0 . \mathrm{CO} ; 2$.

tion model-Formulation. J. Atmos. Sci., 57, 1741-1766, https://doi.org/10.1175/1520-0469(2000)057<1741:AQETCM> 2.0.CO;2.

Protat, A., and Coauthors, 2014: Reconciling ground-based and space-based estimates of the frequency of occurrence and radiative effect of clouds around Darwin, Australia. J. Appl. Meteor. Climatol., 53, 456-478, https://doi.org/10.1175/JAMCD-13-072.1.

Raymond, D. J., 2001: A new model of the Madden-Julian oscillation. J. Atmos. Sci., 58, 2807-2819, https://doi.org/10.1175/ 1520-0469(2001)058<2807:ANMOTM>2.0.CO;2.

— and Ž. Fuchs, 2009: Moisture modes and the Madden-Julian oscillation. J. Climate, 22, 3031-3046, https://doi.org/10.1175/ 2008JCLI2739.1.

- S. L. Sessions, A. H. Sobel, and Ž. Fuchs, 2009: The mechanics of gross moist stability. J. Adv. Model. Earth Syst., 1 (9), https://doi.org/10.3894/JAMES.2009.1.9.

Slingo, A., and J. M. Slingo, 1988: The response of a general circulation model to cloud longwave radiative forcing. I: Introduction and initial experiments. Quart. J. Roy. Meteor. Soc., 114, 1027-1062, https://doi.org/10.1002/ qj.49711448209.
Sobel, A., and C. S. Bretherton, 2000: Modeling tropical precipitation in a single column. J. Climate, 13, 4378-4392, https://doi.org/ 10.1175/1520-0442(2000)013<4378:MTPIAS>2.0.CO;2.

- S. Wang, and D. Kim, 2014: Moist static energy budget of the MJO during DYNAMO. J. Atmos. Sci., 71, 4276-4291, https:// doi.org/10.1175/JAS-D-14-0052.1.

Soden, B. J., A. J. Broccoli, and R. Hemler, 2004: On the use of cloud forcing to estimate cloud feedback. J. Climate, 17, 3661-3665, https://doi.org/10.1175/1520-0442(2004)017<3661: OTUOCF $>2.0 . \mathrm{CO} ; 2$.

Sohn, B. J., T. Nakajima, M. Satoh, and H.-S. Jang, 2010: Impact of different definitions of clear-sky flux on the determination of longwave cloud radiative forcing: NICAM simulation results. Atmos. Chem. Phys., 10, 11 641-11 646, https://doi.org/ 10.5194/acp-10-11641-2010.

Sugiyama, M., 2009: The moisture mode in the quasi-equilibrium tropical circulation model. Part I: Analysis based on the weak temperature gradient approximation. J. Atmos. Sci., 66, 1507-1523, https://doi.org/10.1175/2008JAS2690.1.

Tromeur, E., and W. B. Rossow, 2010: Interaction of tropical deep convection with the large-scale circulation in the MJO.J. Climate, 23, 1837-1853, https://doi.org/10.1175/2009JCLI3240.1.

Wheeler, M. C., and H. H. Hendon, 2004: An all-season real-time multivariate MJO index: Development of an index for monitoring and prediction. Mon. Wea. Rev., 132, 1917-1932, https://doi.org/ 10.1175/1520-0493(2004)132<1917:AARMMI > 2.0.CO;2.

Wielicki, B. A., B. R. Barkstrom, E. F. Harrison, R. B. Lee, G. L. Smith, and J. E. Cooper, 1996: Clouds and the Earth's Radiant Energy System (CERES): An Earth observing system experiment. Bull. Amer. Meteor. Soc., 77, 853-868, https://doi.org/ 10.1175/1520-0477(1996)077<0853:CATERE >2.0.CO;2.

Yasunaga, K., and B. Mapes, 2012: Differences between more divergent and more rotational types of convectively coupled equatorial waves. Part II: Composite analysis based on spacetime filtering. J. Atmos. Sci., 69, 17-34, https://doi.org/10.1175/ JAS-D-11-034.1.

Yoneyama, J. D., C. Zhang, and C. N. Long, 2013: Tracking pulses of the Madden-Julian oscillation. Bull. Amer. Meteor. Soc., 94, 1871-1891, https://doi.org/10.1175/BAMS-D-12-00157.1. 